\title{
Prediction of Hydraulic Automatic Transmission Reliability Using Failure Data Based on Exponential Decay Oscillation Distribution Model
}

\author{
Yongming Liu, ${ }^{1,2}$ Guowen Ye, ${ }^{1,3}$ and Zhuanzhe Zhao $\mathbb{D}^{1,3}$ \\ ${ }^{1}$ School of Mechanical Engineering, Anhui Polytechnic University, Wuhu, Anhui 241000, China \\ ${ }^{2}$ Anhui Provincial Engineering Laboratory on Information Fusion and Control of Intelligent Robot, Wuhu, Anhui 241002, China \\ ${ }^{3}$ Anhui New ReD Institutions of Human-Machine Interaction and Collaboration, Wuhu, Anhui 241000, China \\ Correspondence should be addressed to Zhuanzhe Zhao; zhuanzhe727@ahpu.edu.cn
}

Received 3 July 2020; Revised 4 March 2021; Accepted 29 March 2021; Published 16 April 2021

Academic Editor: Antonio Gloria

Copyright (C) 2021 Yongming Liu et al. This is an open access article distributed under the Creative Commons Attribution License, which permits unrestricted use, distribution, and reproduction in any medium, provided the original work is properly cited.

\begin{abstract}
Aiming at the problem that the traditional reliability models of mechanical products are used to predict the reliability of hydraulic automatic transmission and the expected result is relatively large, firstly, the empirical distribution model line is used to statistically analyze the failure distribution law of the hydraulic automatic transmission; then, the Fourier transform is used to perform frequency domain analysis on experience distribution; on this basis, comprehensively consider the characteristics of experience distribution and frequency domain characteristics of experience distribution, constructs the reliability model of exponential decay oscillation distribution and the corresponding reliability, failure efficiency and average life calculation model; meanwhile, studies the influence of attenuation coefficient, oscillation amplitude, oscillation angle frequency, and other parameters on the probability distribution characteristics. On this basis, the established probability distribution models are adopted to fit the failure time data of hydraulic automatic gearbox carried by a forklift, and the fitting results are compared with exponential distribution models, three-parameter Weibull models, and "bathtub curve" models. The comparing results show that the established exponential decayed oscillation distribution model can better describe the probability distribution characteristics of the fault-free working time of automatic transmission, and the use of this model can obtain a smaller root mean square error. Simultaneously, the research conclusions of this paper can provide meaningful guidance and reference for the analysis of the life distribution model of mechanical products with exponentially attenuated oscillation probability density change law.
\end{abstract}

\section{Introduction}

Product reliability reflects the attributes of product life, failure-free, usability, economy, and so on. The concept of "reliability" was first proposed by the German engineer Lusser, who has been known as the father of reliability. It originated from the theory of how to use probability and mathematical statistics to evaluate the life or failure rate of electronic equipment. In 1943, Lusser proposed a system reliability calculation method to estimate the reliability of the V-2 rocket navigation system. In 1949, the American Society of Radio Engineers established the Reliability Technology Group, which announced the birth of the first reliability professional academic organization.
In August 1952, the U.S. Department of Defense established the Advisory Group on Reliability of Electronic Equipment (AGREE) composed of a group of military, industrial, and academic organizations to investigate, analyze, and study reliability issues. In April 1957, AGREE published the research report "Reliability of Military Electronic Equipment" [1], which elaborated the reliability design, test, and analysis as well as management procedures and methods, and gave the definition of reliability for the first time: "The ability of a product to complete the specified functions under the specified conditions and within the specified time." This report is recognized as the foundation of reliability engineering theory and practice, and it indicates the development direction of reliability engineering. By the 
end of the 1950s, reliability gradually developed into a new science, forming three independent disciplines of reliability mathematics, reliability physics, and reliability engineering, which were increasingly widely used in engineering practice.

When it comes to the complex systems that need to consider fault-related dynamic behaviors, modeling and analysis methods have been continuously studied at home and abroad. Typical methods include dynamic fault trees, stochastic Petri nets, Markov models, and state machine models. However, these modeling methods are generally more complicated and not highly related to system design. At the same time, as a highly abstract method, it is difficult to form a unified modeling standard, so their application scope is restricted. Borzea and Comeaga evaluated the extent to which the simplified Euler-Bernoulli mathematical model can be used to evaluate the parameters of piezoelectric layered structures. A generalized Euler-Bernoulli model is proposed, which is applied to composite beam structures with integrated piezoelectric layers [2]. Based on Info-Gap theory, Kunfeng and Zichun introduced fuzzy parameters to the Minkowski norm and proposed the fuzzy Minkowski norm Info-Gap model. Then the model was applied to InfoGap reliability analysis and proposed a structure-based fuzzy Info-Gap reliability model. Besides, the calculation method of the reliability index was also studied by utilizing fuzzy set theory. The new fuzzy Info-Gap model integrated the interval convex model and ellipsoidal convex model. The new reliability model solved the inconsistencies between two convex models with nonprobabilistic structural reliability analysis. Numerical examples have verified the rationality and applicability of the proposed method [3]. According to Qiao et al., random variable-mass systems have received widespread attention and shown prominent significance with the explosive development of microtechnology and nanotechnologies, so there is a growing need to study the influences of mass disturbance on the systems. The potential failure mode considered is the adhesion failure. First, the original system is approximately replaced by a system without mass disturbance based on an equivalent transformation. Then, the random average and the first-pass time diffusion process theory are combined to obtain the firstpass failure index of the approximate system. The comparisons between the analytical results and those from numerical simulation show that the proposed method works very well. The effects of mass disturbance on the system reliability are explored adequately. It turns out that the presence of mass disturbance can lead to a deterioration of the resonators' reliability severely. It is remarkable that the system has multiple resonance regions with different intensities, and the reliability of the system in the case of primary resonance is much lower than that in the nonresonant case [4]. Yu et al. put forward a reliability cover which is set on at least one of the integrated circuit packages and a Si die of the integrated circuit package. The integrated circuit package is mountable to a printed circuit board via a plurality of solder balls. The reliability cover is configured to reduce a difference in a coefficient of thermal expansion between the integrated circuit package and the printed circuit board and between the Si die and a substrate of the integrated circuit package by a threshold value [5]. Liao et al. believed that many variables affect the sealing performance, and their distribution characteristics are difficult to obtain with probabilistic methods owing to the high cost involved. Numerous problems in engineering are similar due to the appearance of small-sample parameters. In this study, the sealing reliability of an aviation seal was defined as the research object, and an interval uncertainty method and multidimensional response surface were proposed to calculate the sealing reliability. Based on this, the paper first analyzed the failure mechanism of the aviation seal and established a leakage rate model. Then, based on the nonprobabilistic interval model, an interval uncertainty method was proposed to construct the analytical model. With reference to the limit state equation from the structural reliability theory, the multidimensional response surface was used for fast calculation. Afterwards, the single-cylinder gas steering gear in aircraft is chosen as the case study; its sealing reliability in working and nonworking statuses were calculated, and the results were verified with the actual maintenance records. By analyzing the sensitivity of some variables, the surface roughness can be improved only if the cost permits, thereby improving the sealing reliability of the aviation seal. The method proposed in this study realizes the application of small-sample uncertainty analysis in reliability analysis and could provide a feasible way to solve the similar problems in engineering with multidimensional and small-sample parameters [6].

Reference [7] used a time-dependent component state model to describe the trend of component failure rate with component life but does not consider the influence of other factors on component failure rate. References $[8,9]$ analyzed the influence of real-time operating conditions on the reliability model of components for the power system, classified and modeled each outage factor separately, and considered that each outage factor is mutually exclusive. There are limitations to the combined influence of factors on component failure rates. Reference [10] summarized commonly used short-term transmission line reliability models and short-term generator reliability models. Reference [11] used the weighted average method to calculate the average failure rate of the system based on the weather three-state model. Reference [12] used fuzzy technology to establish a weather-related fuzzy component failure rate model. Reference [13] used a decision tree as a single learner and analyzed the influence of environmental parameters on component reliability parameters based on integrated learning. In addition, [14] proposed a gas pipeline failure model related to the degree of corrosion and pipeline air pressure to analyze the failure rate of the pipeline during its life cycle. Reference [15] established a strength failure probability model for heating pipes. Reference [16] proposed a fuel cell fault diagnosis method based on support vector machine (SVM), which predicted its operating status by analyzing the monitoring of fuel cell operating status. Reference [17] analyzed the main fault types of centrifugal compressors and proposed an intelligent fault diagnosis algorithm based on ant colony algorithm. In general, the component modeling for operational reliability assessment 
has a certain research foundation, but the existing research focuses on modeling the individual factors that affect the probability of component state, and its applicability is limited. However, the component modeling of other energy supply systems is mostly focused on individual equipment, and the impact of the external environment is less considered [18].

Life distribution is an important method to describe the distribution law and reliability of failure data of mechanical products and electronic products. It has been widely used in life data analysis, reliability design, fatigue reliability analysis, maintenance decision-making, warranty strategy formulation, and so on $[19,20]$. The accuracy of life distribution decision has a direct impact on product reliability analysis, service life assessment, risk assessment, and maintenance decision-making.

Currently, there are three technical approaches to obtain the life distribution of mechanical product reliability: statistical inference based on test data, modeling based on failure mechanism, and selection of distribution function based on engineering experience.

Based on experimental data, there exist descriptive statistics, nonparametric statistics, parametric statistics, and Bayesian statistics to estimate reliability life distribution and model parameters. Among them, parameter statistics is mostly used in engineering. It is mainly based on the threeparameter Weibull distribution model developed by Weibull et al. [21, 22]. This model can describe the early fault, accidental fault, and wear fault, so it is suitable for the fault distribution of complex equipment and widely used in the field of reliability. Over the years, scholars at home and abroad have studied the three-parameter Weibull model from different perspectives [23-30]. Cordeiro et al. [23] proposed a new exponential Weibull distribution model and studied the probability density function and failure efficiency function of the model. Wang et al. [24] proposed a rolling bearing reliability assessment method based on nuclear principal component analysis and the Weibull proportional risk model, which reduced the differences in manufacturing, installation, and working conditions of the same type of bearings in reliability assessment. Freels et al. [25] proposed several modification methods of two-parameter Weibull distribution to analyze the "bathtub" type life distribution. Baloui [26] established a fuzzy reliability function based on fuzzy Weibull and component life. Nan et al. [27] proposed using Monte Carlo method combined with the maximum likelihood estimation method to perform numerical solution of parameter point estimation for the three-parameter Weibull distribution model. Linying et al. [28], based on exponential Weibull distribution, proposed a reliability modeling method for complex electronic systems, which can dynamically describe the changes in system reliability. González et al. [29] proposed a reliability model for describing the lifetime of electronic devices with parametric functions under time-varying voltages. The model is based on stochastic failure rate cumulative damage model and modified power reciprocal law. In order to estimate the parameters of the model, the maximum likelihood method is used. Mendez-Gonzalez et al. [30] proposed a model that incorporates power harmonics into the power-law model. Based on the cumulative damage model, the time-varying voltage is modeled as a power-law model by using the compact form of the Fourier series.

Due to the limitation of the development cost, cycle time, test analysis technology, and so on, failure test data are usually insufficient to accurately infer life distribution. In order to make full use of expert experience, historical data, and other pieces of information to solve the problem of insufficient experimental data, nonparametric methods and Bayesian methods have attracted more and more attention. The combination of classical statistical analysis method and Bayesian statistical analysis methods can improve the estimation accuracy of parameters to some extent. The core and key of the Bayesian analysis method is how to use prior information to determine the prior distribution. Literature [31] proposed that, according to the maximum entropy principle, all kinds of empirical information could be regarded as different constraint conditions, and the optimal empirical distribution could be determined by maximizing entropy. However, the Bayesian statistical inference method is very dependent on the prior distribution, and there is no general method to confirm and testing the prior distribution.

In order to infer more accurate life distribution, it is necessary to combine product failure mechanism, test data, and engineering experience to conduct inferential analysis from both statistical and verification aspects [32, 33]. Literature [34] constructed a method to test multiple failure physics equations and cumulative damage criteria under Weibull distribution and timed truncation test from the aspects of failure physics, hypothesis testing principle, regression theory, and so on, but its effectiveness lacks the support of the strict statistical theory.

As for the statistical analysis of accelerated life test data, Park and Bae [35] proposed a method to derive life distribution by using product degradation data. The disadvantage of this method is that it relies too much on the researcher's experience in determining the distribution of the random parameters.

The degradation trajectory model cannot express the uncertainty of product performance degradation over time. In order to solve this problem, the Winer stochastic process model [36-39], the Gamma stochastic process model $[40,41]$, the inverse Gaussian stochastic process model [42-44], the compound Poisson stochastic process model [45], and so on have gradually been used to accelerate degradation data analysis.

The life distribution corresponding to the stochastic process model can be obtained by theoretical derivation, and the methods mainly include approximation method, analytical method, and numerical method. The approximation method extrapolates the pseudofailure life by fitting the degradation trajectory of each sample and then matches the life distribution according to this fitting method. However, this method ignores the uncertainty and measurement error in the pseudofailure life prediction, and there is a deviation between the fitted-life distribution and the real-life distribution. The life distribution can be derived directly from the distribution of the parameters of the degradation model by 
the analytical method, but this method is only applicable to the case where there is only one random parameter and the parameter distribution is known. For example, in the linear Wiener process, the life distribution can be derived as an inverse Gaussian distribution based on the type of failure time $[36,37]$; for the standard Gamma process, the life distribution can be derived as the BS distribution (Birnbaum-Saunders distribution) [46]. When the performance degradation model adopts a complex stochastic process (such as a nonlinear trajectory model that includes multiple random parameters), numerical methods are used to sample the parameter estimates of the degradation model to infer the life distribution. For example, regarding the nonlinear Wiener process, Si et al. [47] provided an approximate solution method for the life distribution. In short, the analytical method can be used to obtain the analytical expression of the life distribution, while the use of the approximate method and the numerical method requires the use of pseudofailure life to estimate the empirical distribution of product life.

Nouguier et al. discussed the validity of the existing cumulative distribution function (CDF) formulas for the defect centric model (DCM), especially in the low defect number regime (defect number $<3$ ). After finding their limitations, this paper proposes an improved DCM CDF formula, which well illustrates the low defect count scheme and becomes very crucial in advanced CMOS technology with scalable devices. The benefits of the revisited DCM CDF formula are experimentally demonstrated on PMOS devices from $28 \mathrm{~nm}$ FDSOI technology subjected to BTI stress as well as on Monte Carlo simulated Vth data [48]. Peng et al. proposed an improved centered cycle method for identifying the Preisach distribution function based on the nonuniform discretization. The principle and the limits of the centered cycle method were first introduced. Then, the nonuniform discretization method was put forth based on the curvature of the unmagnetized curve. The numbers of loops for identifying the distribution function are decided by the proportion of the integral of curvature. Also, the method to determine the cell values of the nonuniformly discrete Preisach triangle was introduced. The proposed method was validated by the laboratory test of a toroidal sample. The results show that the precision of the proposed method for predicting the hysteresis loop is higher than the centered cycle method by the same number of loops used for identifying [49]. Lin et al. proposed a modulation classification algorithm that is based on the received signal's amplitude for coherent optical receivers. The proposed algorithm classifies the modulation format from several possible candidates by differentiating the CDF curves of their normalized amplitudes. The candidate with the most similar CDF to the received signal is selected. The measure of similarity is the minimum average distance between these CDFs. Five commonly used quadrature amplitude modulation formats in digital coherent optical systems are employed. Optical back-to-back experiments and extended simulations are carried out to investigate the performance of the proposed algorithm. Results show that the proposed algorithm achieves accurate classification at optical signal-to-noise ratios of interest. Furthermore, it does not require carrier recovery [50].

The parameter estimation method of the model is related to the characteristics of the model and the types of data; the most extensive method is the very large likelihood estimation method. Ye et al. [51] gave the parameter maximum likelihood estimation method for the Wiener process model with consideration of error and the Winer process model with consideration of random effect. In addition to using the maximum likelihood estimation method, the linear regression property of the degradation trajectory can also be used to estimate the parameters of the model by using the least square estimation method, such as the literature [52-54] adopted this method.

Commonly used methods for solving parameter estimation models include exhaustive method, search algorithm, genetic algorithm, and expectation maximization (EM) algorithm. Hao et al. [38] considered the influence of test error and stress error and used genetic algorithm to estimate the parameters of the Wiener process model. Ye et al. [51] used the EM algorithm to solve the likelihood function and obtained the estimated values of the parameters of the inverse Gaussian process model.

Generally speaking, inferred product life distribution is still one of the main technical bottlenecks affecting the development and application of reliability life test technology, especially for comprehensive stress accelerated life test. The slight deviation in life distribution models may lead to serious errors in the reliability assessment conclusions.

For some products with complex structure and working environment, the life distribution and failure physical equation are difficult to described by simple models. Particularly, breakthroughs should be made in information integration, inference, and verification of prior distribution. Meanwhile, the mechanical product is usually a relatively complex system, and field data can best reflect its true reliability level. If the reliability data in the field use process can be combined with the reliability test, the reliability evaluation model is established first, and then the accelerated test data are used to estimate the model parameters, which will help reduce the test sample size.

In engineering practice, many failures of complex products are periodic and oscillating, and their life distribution will show the change rule of attenuation oscillations. With the increase of service time, the probability density of the product life distribution decays exponentially and oscillates locally. At present, most researches on the Weibull model at home and abroad ignore the oscillation of probability density caused by the periodic failure of mechanical products, which often has some errors with actual engineering data and affects the accuracy of the model application.

This paper is organized as follows. In Section 2, first, the empirical distribution model line is used to analyze the statistical characteristics of the failure distribution of the hydraulic automatic transmission; the Fourier transform is used to analyze the empirical distribution in the frequency domain. In Section 3, on this basis, the empirical distribution characteristics and the empirical distribution frequency 
domain are comprehensively considered a feature, constructed a life distribution model with the probability density distribution law of exponential decay oscillation, and gave the corresponding calculation models of reliability, failure efficiency, and average life. In Section 4, the attenuation coefficient, oscillation amplitude, oscillation angle frequency, and other parameters on the probability distribution characteristics are studied. Section 5 presents research on the influence of different parameters on the sensitivity of life probability distribution characteristics. Section 6 establishes parameter estimation model by using the least square method. In Section 7, finally, the failure time data of the hydraulic automatic gearbox carried by a certain type of forklift are taken as the research object, and the established probability distribution model is used. The failure time data of this type of hydraulic automatic gearbox is fitted and compared with the fitting results of the exponential distribution model, three-parameter Weibull model, and "bathtub curve" model to verify the validity of the established probability distribution model.

\section{Analysis of Hydraulic Automatic Transmission Reliability Characteristics}

2.1. Failure Data and Failure Diagram. In order to verify the practicability of the model, a forklift truck is taken as an example. This type of forklift was sold 30,000 units in 2018, and the after-sales maintenance records have been tracked for one year. Its 117 hydraulic automatic transmissions equipped with the instrument panel failed, and part of the failure time recorded by the instrument panel was arranged in ascending order, as shown in Table 1.

The disassembly process is shown in Figure 1.

Parts of the faulty parts are shown in Figure 2 (the fault location in the figure is circled in red).

The fault location in the figure is circled in red.

2.2. Statistical Characteristic of the Hydraulic Automatic Transmission Reliability Based on Empirical Distribution. Adopting the method of empirical distribution, the reliability curve of the failure time of the hydraulic gearbox is figured out with $\Delta t=211.033(n=9)$. The cumulative failure probability distribution of empirical distribution is linearly interpolated, and the probability density distribution curve and the failure rate curve of the failure time of the hydraulic gearbox are calculated as shown in Figures 3-5.

It can be seen from Figures $3-5$ that the life probability density and reliability of the hydraulic automatic gearbox exhibit damped oscillation characteristics as time increases.

The changes of empirical distribution failure probability density, reliability, and failure rate with $\Delta t$ (the interval is divided into $n$ sections) are shown in Figures 6-8.

It can be seen from Figures 6-8 that the empirical distribution failure probability density, reliability, and failure rate vary with $\Delta t$. The first half of the curve shows the
TABLE 1: Failure time of automatic transmission.

\begin{tabular}{|c|c|c|c|}
\hline Number & Failure time $(\mathrm{h})$ & Number & Failure time (h) \\
\hline 1 & 0.7 & 2 & 5 \\
\hline 3 & 10 & 4 & 15 \\
\hline 5 & 18 & 6 & 23.8 \\
\hline 7 & 30 & 8 & 35 \\
\hline 9 & 39.3 & 10 & 48 \\
\hline 11 & 56.8 & 12 & 60 \\
\hline 13 & 62 & 14 & 90 \\
\hline 15 & 95.3 & 16 & 107.2 \\
\hline 17 & 110.7 & 18 & 112 \\
\hline 19 & 116.5 & 20 & 131.8 \\
\hline 21 & 134.7 & 22 & 135 \\
\hline 23 & 147 & 24 & 156 \\
\hline 25 & 166.9 & 26 & 213 \\
\hline 27 & 218.1 & 28 & 227.1 \\
\hline 29 & 234.3 & 30 & 240 \\
\hline 31 & 247 & 32 & 250 \\
\hline 33 & 259 & 34 & 260 \\
\hline 35 & 281 & 36 & 285 \\
\hline 37 & 300 & 38 & 320 \\
\hline 39 & 326 & 40 & 330 \\
\hline 41 & 338 & 42 & 350 \\
\hline 43 & 360 & 44 & 360.8 \\
\hline 45 & 370 & 46 & 390 \\
\hline 47 & 413 & 48 & 430 \\
\hline 49 & 468.1 & 50 & 477.1 \\
\hline 51 & 484 & 52 & 500 \\
\hline 53 & 510 & 54 & 512 \\
\hline 55 & 520 & 56 & 523 \\
\hline 57 & 560 & 58 & 570 \\
\hline 59 & 576 & 60 & 577 \\
\hline 61 & 590 & 62 & 597 \\
\hline 63 & 600 & 64 & 623 \\
\hline 65 & 630 & 66 & 630 \\
\hline 67 & 670 & 68 & 685 \\
\hline 69 & 709 & 70 & 739.3 \\
\hline 71 & 748 & 72 & 756.8 \\
\hline 73 & 760 & 74 & 773.1 \\
\hline 75 & 780 & 76 & 795.3 \\
\hline 77 & 810.7 & 78 & 812 \\
\hline 79 & 860 & 80 & 886 \\
\hline 81 & 901 & 82 & 932.4 \\
\hline 83 & 932.4 & 84 & 938 \\
\hline 85 & 948 & 86 & 971 \\
\hline 87 & 980 & 88 & 980 \\
\hline 89 & 1026.6 & 90 & 1040 \\
\hline 91 & 1051.6 & 92 & 1107 \\
\hline 93 & 1132 & 94 & 1155 \\
\hline 95 & 1207 & 96 & 1218.1 \\
\hline 97 & 1223 & 98 & 1232 \\
\hline 99 & 1265 & 100 & 1282 \\
\hline 101 & 1312 & 102 & 1350 \\
\hline 103 & 1360 & 104 & 1362.2 \\
\hline 105 & 1386 & 106 & 1397 \\
\hline 107 & 1476 & 108 & 1490 \\
\hline 109 & 1556 & 110 & 1576 \\
\hline 111 & 1613 & 112 & 1638 \\
\hline 113 & 1660.8 & 114 & 1690 \\
\hline 115 & 1813 & 116 & 1862 \\
\hline
\end{tabular}




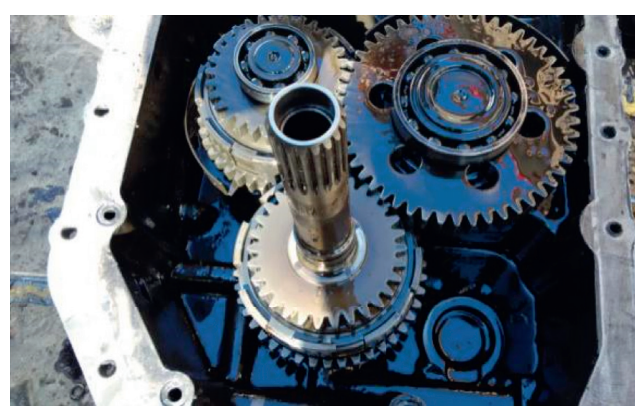

(a)

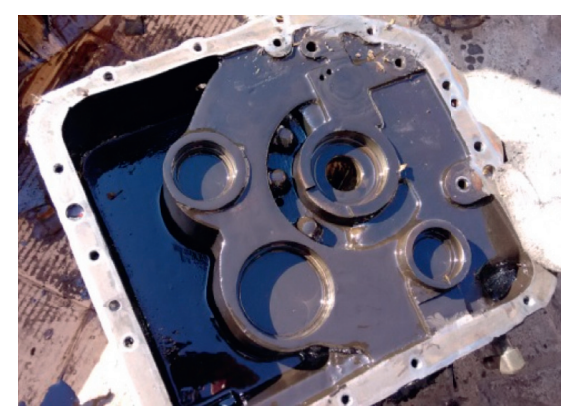

(b)

Figure 1: Disassembly process diagram.

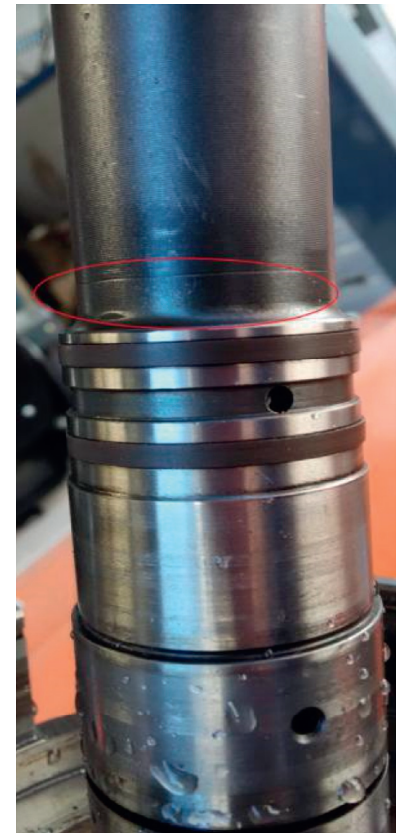

(a)

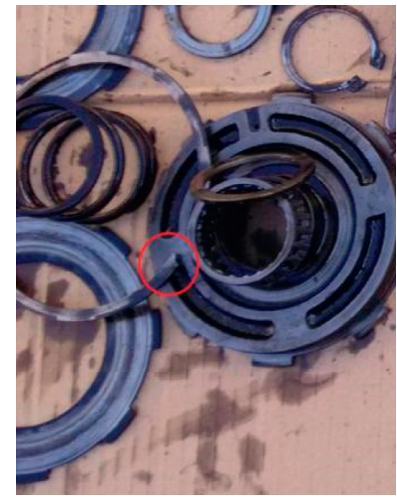

(c)

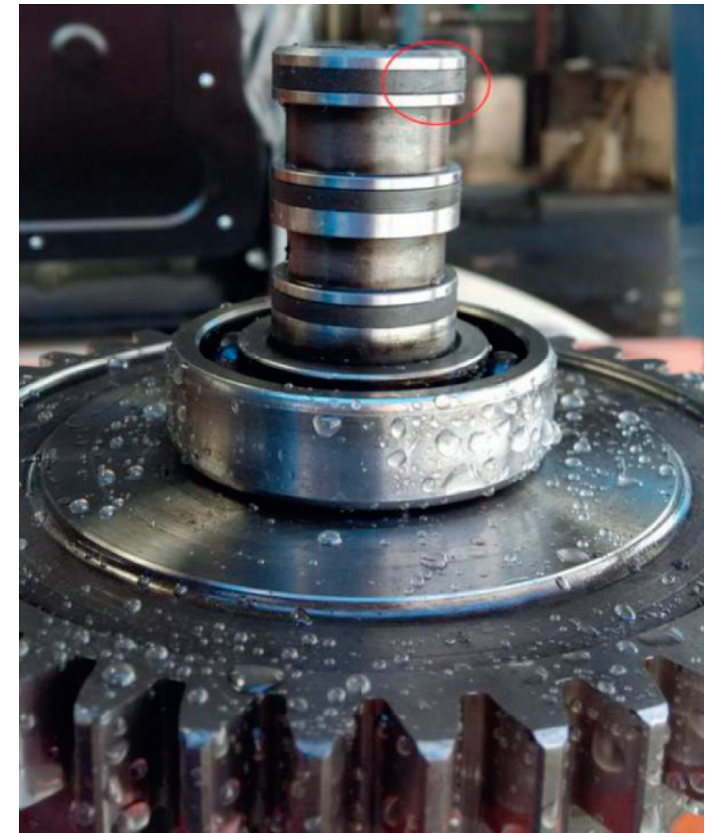

(b)

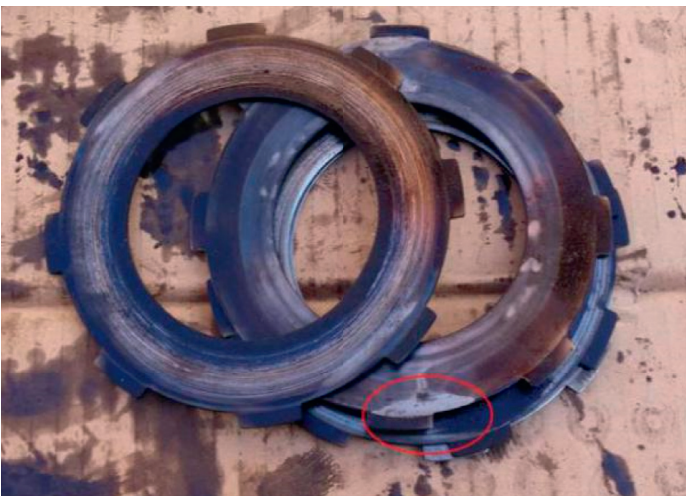

(d)

Figure 2: Partially failed parts.

characteristics of attenuated oscillation, while the second half of the curve remains unchanged. Using the method of empirical distribution, it can be seen that the reliability model of the hydraulic automatic transmission has the characteristics of damping oscillation, but the frequency domain characteristics of the reliability model cannot be accurately judged. That is because the theoretical calculation formula of probability density is 


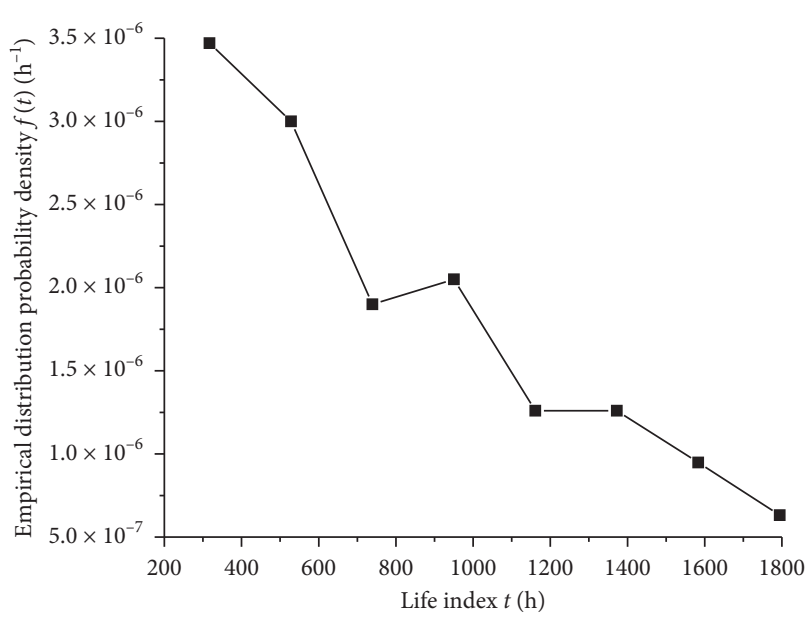

FIgure 3: Probability density of empirical distribution.

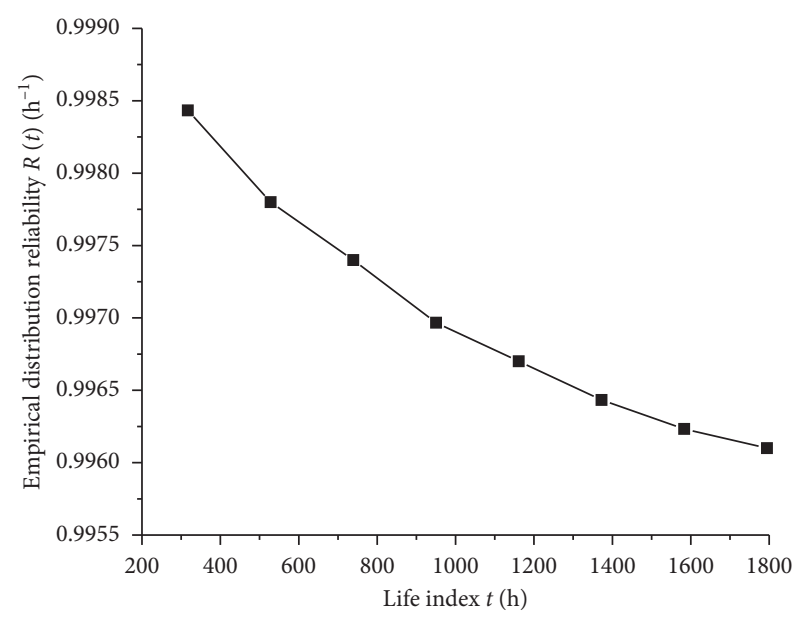

FIGURE 4: Reliability of empirical distribution.

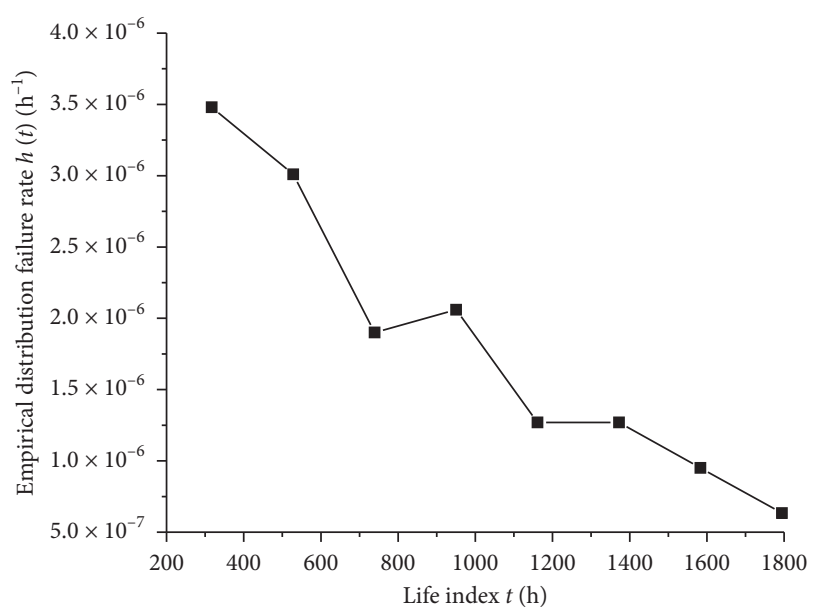

Figure 5: Failure rate of empirical distribution.

$$
f(t)=\lim _{\Delta t \longrightarrow 0} \frac{F(t+\Delta t)-F(t)}{\Delta t}=F^{\prime}(t) .
$$

It is the process of taking the limit of the increment $\Delta F$ of the cumulative failure probability to the time increment $\Delta t$ and the process of taking the limit, which requires the time increment to be infinitely small. Also, it is the derivative of the cumulative failure probability distribution function $F$ to the random variable $t$. When using the empirical distribution model to calculate the probability density, the firstorder forward difference numerical calculation method is adopted. Since the time increment $\Delta t$ is a finite value, and due to the small amount of data, usually the random variable is divided into $8 \sim 10$ intervals when calculating the cumulative failure probability of the empirical distribution. What is more, because the time is $t \in[0.7,1900]$ and the value is large, $\Delta t \in[189.93,237.4125]$ when $\Delta t$ adopts the above interval division method. As a result, $\Delta F \in[0.000133$, 0.000733], which is much larger than the infinitesimal and differentiability requirements, and there is a large error in the value. Meanwhile, the cumulative failure distribution function of the empirical distribution is interpolated. Although the cumulative failure distribution function of empirical distribution is continuous, it cannot satisfy the derivability, and there is a large error in calculating the failure rate probability density model.

\subsection{Frequency Characteristic of the Hydraulic Automatic} Transmission Reliability Empirical Distribution. In order to overcome the large numerical error and theoretical error in judging the frequency characteristics of probability density distribution with the empirical distribution method, the Fourier transform method is used to analyze the cumulative failure probability distribution of the empirical distribution.

It can be seen from Figure 9 that the amplitude spectrum of the cumulative failure probability of the empirical distribution has a distinct characteristic frequency, and the oscillation period corresponding to the characteristic frequency is about $T=209.33(\mathrm{~km})$.

Obviously, the cumulative failure probability of the hydraulic automatic gearbox is not easy to use the traditional exponential distribution, normal distribution, two-parameter Weibull distribution, three-parameter Weibull distribution, and other probability density models to describe. Therefore, it is necessary to establish a reliability model that satisfies the exponentially damped oscillation characteristics according to the failure data characteristics of the hydraulic automatic transmission.

\section{Construction of Exponential Decay Oscillation Probability Density Distribution Model}

When the product failure rate curve has an oscillating feature, its probability density has the characteristic of exponentially declining oscillation. The three-parameter product life probability distribution model shown in equation (2) can be constructed, and its probability density function is 


$$
\begin{aligned}
f(t)= & \lambda[A \omega \sin (2 \omega t)+1] e^{-\lambda\left[A \sin ^{2}(\omega t)+t\right]}, \quad \lambda>0,|A \omega| \\
& <1, t \geq 0,
\end{aligned}
$$

where $t$ is the life measurement index and $t \geq 0 ; \lambda$ is the attenuation coefficient; $A$ is the oscillation amplitude; $2 \omega$ is the oscillation angular frequency.

The probability that a product loses a specified function (i.e., a failure) under specified conditions and within a specified time is called the cumulative failure probability (also called unreliability), so the unreliability function $F(t)$ can be regarded as the probability of the event " $T \leq t$, , which is

$$
F(t)=P(T \leq t)=\int_{-\infty}^{t} f(t) \mathrm{d} t .
$$

From the life probability density function shown in equation (2), the cumulative life distribution function of the product can be derived as

$$
\begin{aligned}
F(t) & =\int_{-\infty}^{t} f(t) \mathrm{d} t \\
& =\int_{0}^{t} \lambda[A \omega \sin (2 \omega t)+1] e^{-\lambda\left[A \sin ^{2}(\omega t)+t\right]} \mathrm{d} t \\
& =1-e^{-\lambda\left[A \sin ^{2}(\omega t)+t\right]}, \quad t \geq 0 .
\end{aligned}
$$

Obviously, equation (2) satisfies the following conditions, namely,

$$
\int_{-\infty}^{+\infty} f(t) \mathrm{d} t=\int_{-\infty}^{+\infty} \lambda[A \omega \sin (2 \omega t)+1] e^{-\lambda\left[A \sin ^{2}(\omega t)+t\right]} \mathrm{d} t=1, \quad t \geq 0
$$

At the same time, formula (3) can meet the following completeness requirements, namely,

$$
\begin{aligned}
& F(-\infty)=0, \\
& F(+\infty)=1 .
\end{aligned}
$$

Therefore, equation (2) can be used as the probability density function of life distribution, equation (3) can be used as the CDF of life distribution, and equations (2) and (3) can be used as exponentially decaying oscillation type life distribution models.

Further, the reliability function $R(t)$ can be regarded as the probability of event " $T>t$," according to the relationship between reliability and cumulative life distribution function, the expression of reliability can be derived as

$$
R(t)=1-F(t)=e^{-\lambda\left[A \sin ^{2}(\omega t)+t\right]}, \quad t \geq 0 .
$$

The failure rate $h(t)$ is the conditional probability that a product in a unit time of $t+\Delta t$ has not yet failed at time $t$; namely,

$$
h(t)=\lim _{\Delta t \longrightarrow 0} \frac{P(t<T<((t+\Delta t) / T)>t)}{\Delta t} .
$$

From the nature of the conditional probability formula and the inclusion relationship of time,

$$
\begin{aligned}
P\left(t<T \leq \frac{t+\Delta t}{T}>t\right) & =\frac{P(t<T \leq t+\Delta t)}{P(T>t)}=\frac{F(t+\Delta t)-F(t)}{R(t)}, \\
h(t) & =\lim _{\Delta t \longrightarrow 0} \frac{F(t+\Delta t)-F(t)}{\Delta t} \cdot \frac{1}{R(t)} \\
& =\frac{F^{\prime}(t)}{R(t)}=\frac{f(t)}{R(t)} .
\end{aligned}
$$

Then, the gain and failure rate $h(t)$ can be expressed as

$$
h(t)=\frac{f(t)}{R(t)}=\lambda[A \omega \sin (2 \omega t)+1], \quad t \geq 0 .
$$

The expression of the average life calculation model is

$$
\begin{aligned}
T_{\mathrm{MTBF}} & =E(t)=\int_{0}^{+\infty} t f(t) \mathrm{d} t \\
& =\int_{0}^{+\infty} \lambda t[A \omega \sin (2 \omega t)+1] e^{-\lambda\left[A \sin ^{2}(\omega t)+t\right]} \mathrm{d} t, \quad t \geq 0 .
\end{aligned}
$$

\section{The Study on the Characteristics of Life Probability Distribution with Different Parameters}

According to the probability distribution model shown in equation (2), the effects of attenuation coefficient $\alpha$, oscillation amplitude $A$, and oscillation angular frequency $2 \omega$ on the probability distribution characteristics of life are studied, respectively.

4.1. Influence of Attenuation Coefficient on the Characteristics of Life Probability Distribution. When $A=100, \omega=0.001$, and $\lambda$ is $0.0002,0.0005$, and 0.0008 , respectively, the corresponding probability density curve, reliability change curve, and failure rate change curve are shown in Figures 10-12.

It can be seen from Figures 10 to 12 that the smaller the attenuation coefficient $\lambda$ is, the lower the failure rate is, the higher the reliability is, and the smoother the change in probability density is. Simultaneously, it can be seen from Figure 10 that the probability density exhibits an exponentially declining oscillation change law. Figure 12 shows that the failure rate is no longer an invariant constant, but it has certain periodicity and volatility, and the fluctuation period remains the same.

\subsection{Influence of Oscillation Amplitude on the Characteristics of} Life Probability Distribution. When $A=100, \omega=0.001$, and $A$ is 200,500 , and 800 , respectively, the corresponding probability density curve, reliability change curve, and failure rate change curve are shown in Figures 13-15.

It can be seen from Figures 13 to 15 that the larger the oscillation amplitude $A$ is, the more intense the oscillations of probability density, reliability, and failure rate are. At the same time, it can be seen from Figure 13 that the probability density exhibits an exponentially declining oscillation 


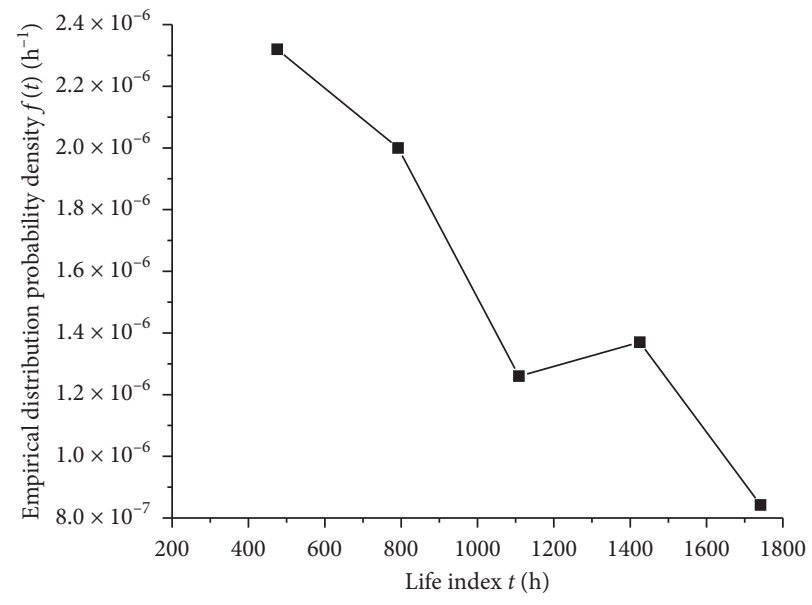

(a)

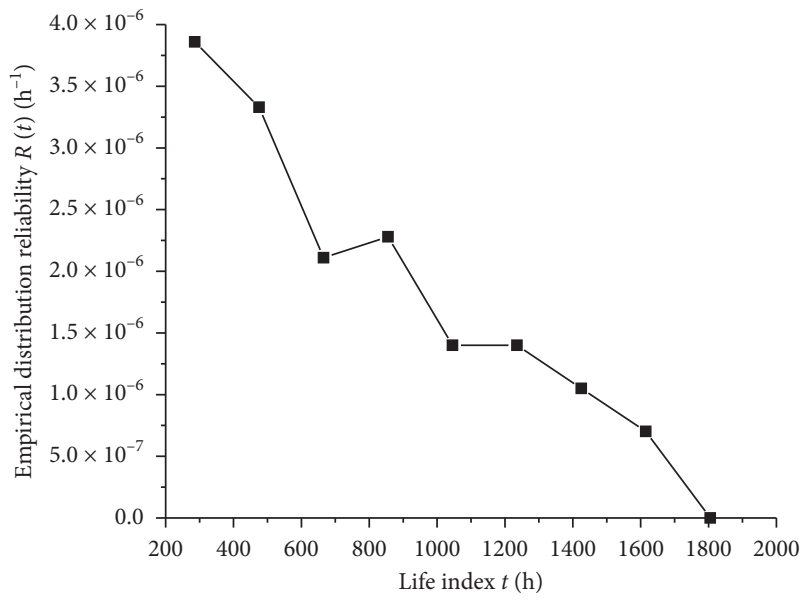

(b)

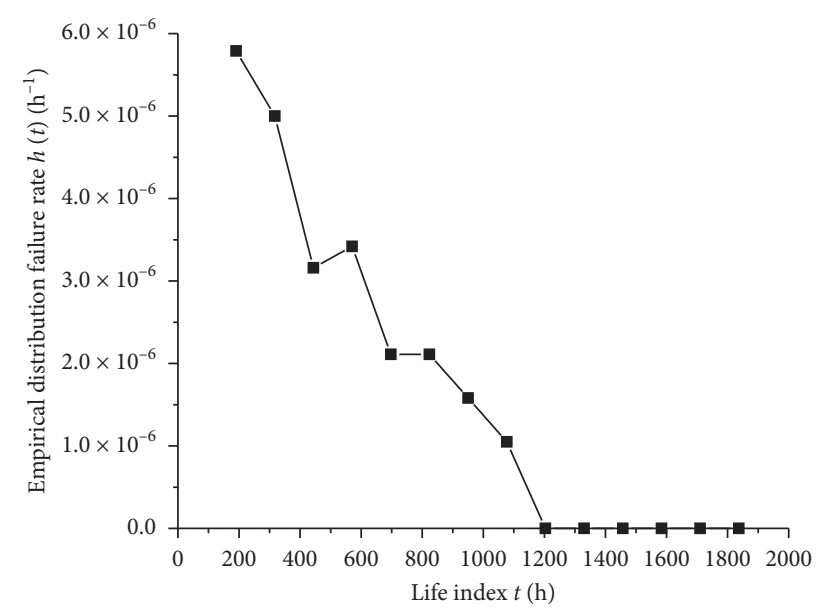

(c)

FiguRe 6: Empirical distribution uncertainty probability density changes with $\Delta t$. (a) $\Delta t=316.55(n=6)$. (b) $\Delta t=189.93$ ( $n=10)$. (c) $\Delta t=126.62(n=15)$.

change law. Figure 15 illustrates that the failure rate is no longer an invariant constant, but it has certain periodicity and volatility, and the fluctuation period is the same.

4.3. Influence of Oscillation Angular Frequency on the Characteristics of Life Probability Distribution. When $\lambda=0.0002$, $A=500$, and $\omega$ is $0.0002,0.0004$, and 0.0006 , respectively, the corresponding probability density curve, reliability change curve, and failure rate change curve are shown in Figures 16-18.

It can be seen from Figures 16-18 that the larger the oscillation angular frequency $\omega$ is, the faster the oscillations of probability density, reliability, and failure rate are. At the same time, it can be seen from Figure 16 that the probability density exhibits an exponentially declining oscillation change law. Figure 18 shows that the failure rate is not an invariant constant, but it has certain periodicity and volatility. In addition, the larger $\omega$ gets, the smaller the fluctuation period becomes.

\section{Research on the Influence of Different Parameters on the Sensitivity of Life Probability Distribution Characteristics}

5.1. Establishment of the Sensitivity Model. Sensitivity analysis of $f(t)$ to $\lambda$ is as follows:

$$
\begin{aligned}
\frac{\partial f(t)}{\partial \lambda}= & -\left[A \sin ^{2}(\omega t)+t\right] \times[A \omega \sin (2 \omega t)+1] \\
& \times e^{-\lambda\left[A \sin ^{2}(\omega t)+t\right]} .
\end{aligned}
$$

Sensitivity analysis of $f(t)$ to $A$ is as follows:

$\frac{\partial f(t)}{\partial A}=-\lambda \sin ^{2}(\omega t) \times \lambda \omega \sin (2 \omega t) \times e^{-\lambda\left[A \sin ^{2}(\omega t)+t\right]}$.

Sensitivity analysis of $f(t)$ to $\omega$ is as follows: 


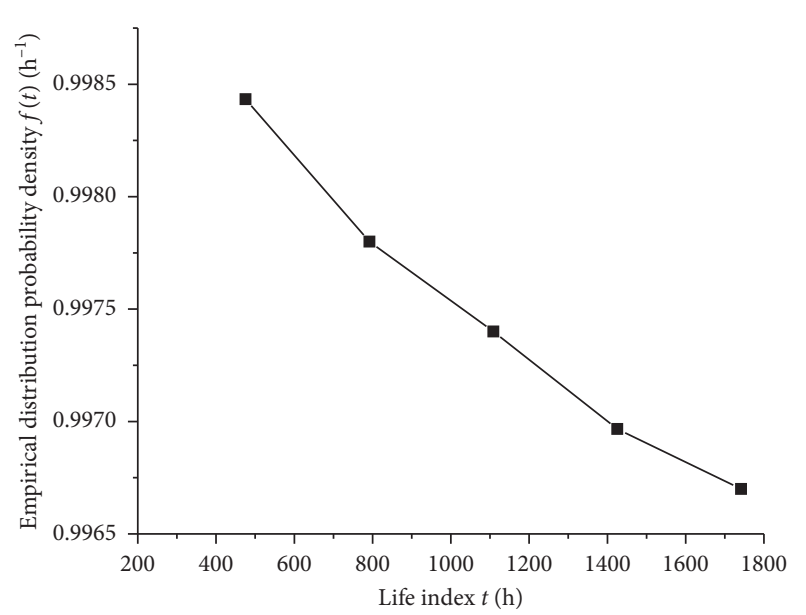

(a)

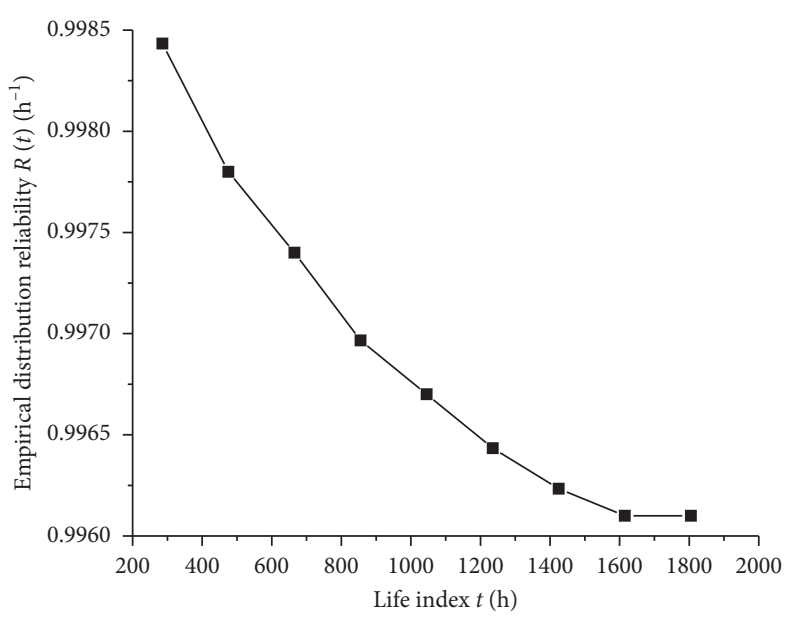

(b)

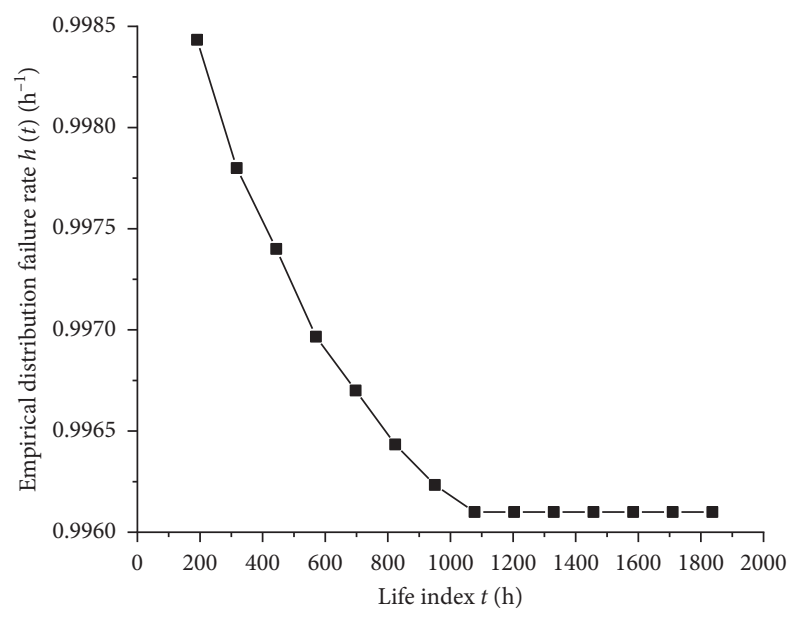

(c)

FIgURE 7: Reliability changes with $\Delta t$. (a) $\Delta t=316.55(n=6)$. (b) $\Delta t=189.93(n=10)$. (c) $\Delta t=126.62(n=15)$.

$$
\begin{aligned}
\frac{\partial f(t)}{\partial \omega}= & -\lambda A t \times \frac{1-\cos (2 \omega t)}{2} \times[\lambda A \sin (2 \omega t) \\
& +2 \lambda \omega A \cos (2 \omega t)] \times e^{-\lambda\left[A \sin ^{2}(\omega t)+t\right]} .
\end{aligned}
$$

Sensitivity analysis of $R(t)$ to $\lambda$ is as follows:

$$
\frac{\partial R(t)}{\partial \lambda}=-\left[A \sin ^{2}(\omega t)+t\right] \times e^{-\lambda\left[A \sin ^{2}(\omega t)+t\right]} .
$$

Sensitivity analysis of $R(t)$ to $A$ is as follows:

$$
\frac{\partial R(t)}{\partial A}=-\lambda \sin ^{2}(\omega t) \times e^{-\lambda\left[A \sin ^{2}(\omega t)+t\right]} .
$$

Sensitivity analysis of $R(t)$ to $\omega$ is as follows:

$$
\frac{\partial R(t)}{\partial \omega}=-\lambda A t \times \frac{1-\cos (2 \omega t)}{2} \times e^{-\lambda\left[A \sin ^{2}(\omega t)+t\right]} .
$$

Sensitivity analysis of $h(t)$ to $\lambda$ is as follows:

$$
\frac{\partial h(t)}{\partial \lambda}=A \omega \sin (2 \omega t)+1
$$

Sensitivity analysis of $h(t)$ to $A$ is as follows:

$$
\frac{\partial h(t)}{\partial A}=\lambda \omega \sin (2 \omega t)
$$

Sensitivity analysis of $h(t)$ to $\omega$ is as follows:

$$
\frac{\partial h(t)}{\partial \omega}=\lambda A \sin (2 \omega t)+2 \lambda A \omega t \cos (2 \omega t) .
$$

In view of the above formula, the influence of attenuation coefficient $\lambda$, oscillation amplitude $A$, and oscillation angular frequency $\omega$ on sensitivity are studied, respectively.

5.2. The Influence of Attenuation Coefficient on the Sensitivity of Life Probability Distribution. When $A=100, \omega=0.001$, and $\lambda$ is $0.0002,0.0005$, and 0.0008 , respectively, the corresponding probability density, reliability, and failure rate versus the sensitivity of the oscillation amplitude $A$ are shown in Figures 19-21.

It can be seen from Figures 19 to 21 that the smaller the attenuation coefficient is, the smaller the sensitivity fluctuation of the failure rate with respect to the oscillation amplitude $A$ is, the lower the sensitivity of the reliability with respect to the oscillation amplitude $A$ is, and the gentler the 


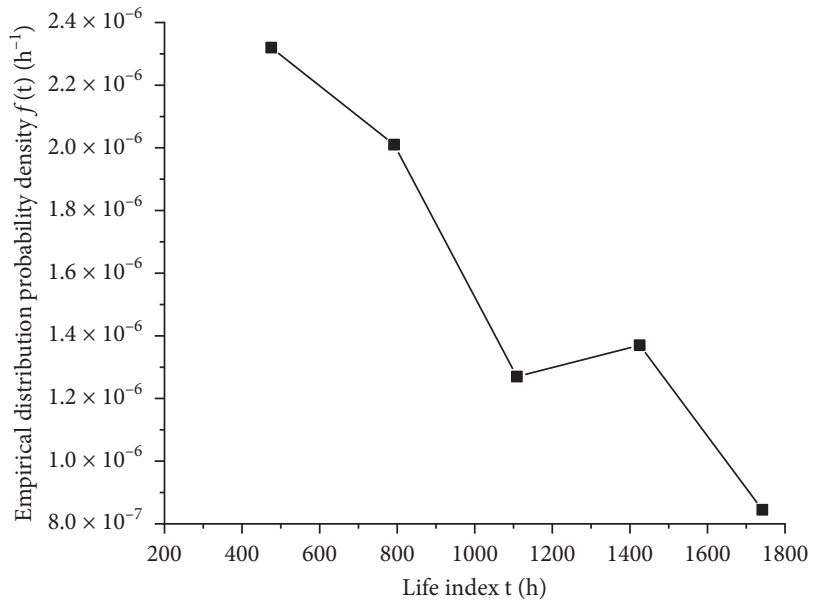

(a)

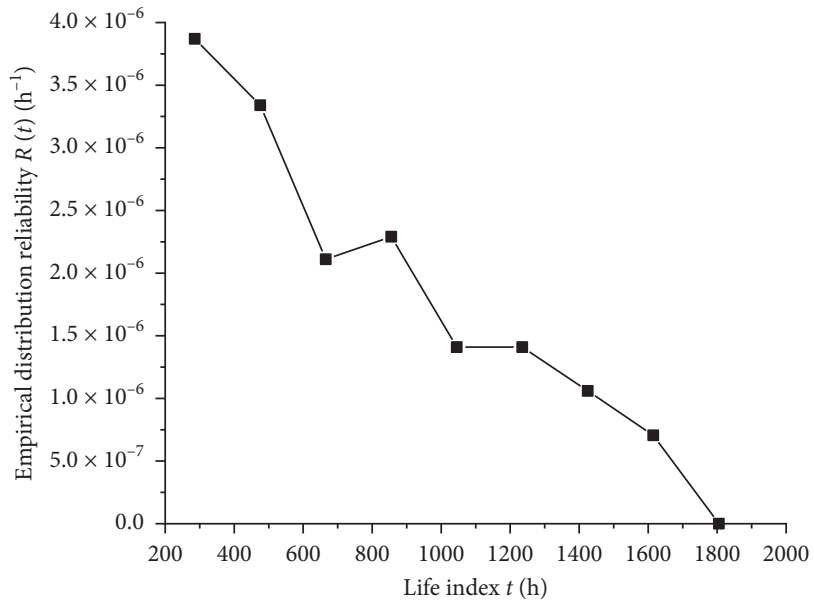

(b)

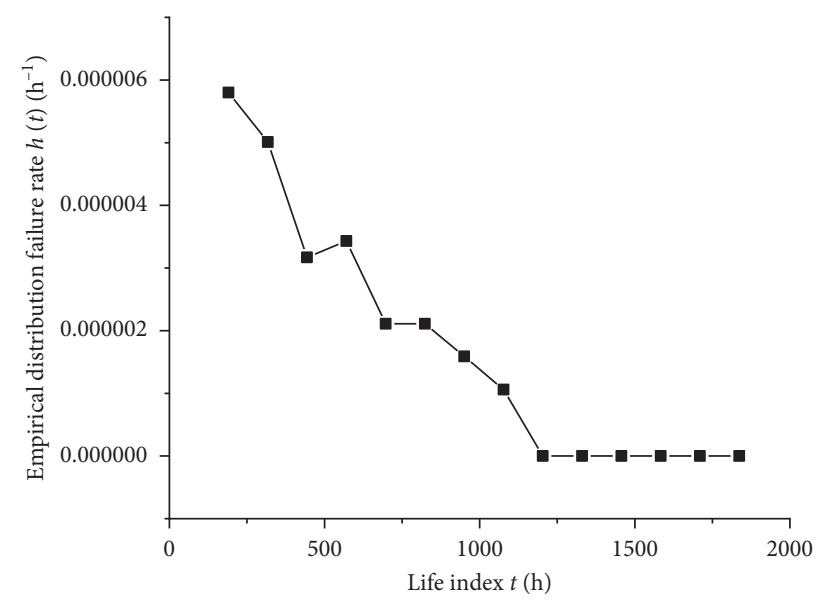

(c)

Figure 8: The failure rate varies with $C$. (a) $\Delta t=316.55(n=6)$. (b) $\Delta t=189.93(n=10)$. (c) $\Delta t=126.62(n=15)$.

sensitivity of the probability density change with respect to the oscillation amplitude $A$ is. At the same time, it can be seen from Figures 19 and 20 that the sensitivity of the probability density with respect to the oscillation amplitude $A$ fluctuates greatly at the beginning, and the fluctuation becomes smaller as it goes later. It can be seen from Figure 21 that the sensitivity change of the failure rate with respect to the oscillation amplitude $A$ has certain periodicity and volatility, and the fluctuation period is the same.

When $A=100, \omega=0.001$, and $\lambda$ is $0.0002,0.0005$, and 0.0008 , respectively, the corresponding variation curves of probability density, reliability, and failure rate with respect to the sensitivity of the oscillation angular frequency $2 \omega$ are shown in Figures 22-24.

It can be seen from Figure 22 that the smaller the attenuation coefficient, the greater the sensitivity fluctuation of the probability density with respect to the oscillation angular frequency $2 \omega$. Also, it can be seen from Figures 23 and 24 that the smaller the attenuation coefficient is, the greater the fluctuation of the failure rate with respect to the oscillation angular frequency $2 \omega$ sensitivity is. The smaller the reliability is, the lower the sensitivity to the oscillation angular frequency $2 \omega$ is. In addition, it can be seen from Figure 6 that the failure rate has a certain periodicity and fluctuation with respect to the sensitivity change of the oscillation angular frequency $2 \omega$, and the fluctuation period is the same.

\subsection{The Influence of Oscillation Amplitude on the Sensitivity of} Life Probability Distribution Characteristics. When $\lambda=0.0002, \omega=0.001$, and $A$ is 200,500 , and 800 , respectively, the corresponding probability density, reliability, and failure rate versus attenuation coefficient $\lambda$ sensitivity change curves are shown in Figures 25-27.

It can be seen from Figures 25-27 that the greater the oscillation amplitude $A$, the greater the value of the sensitivity change. At the same time, it can be seen from Figures 25 and 26 that the probability density changes with respect to the attenuation coefficient $\lambda$ sensitivity and reliability with respect to the attenuation coefficient $\lambda$ sensitivity. Figure 27 shows that the failure rate has a periodicity with respect to the attenuation coefficient $\lambda$ sensitivity.

When $\lambda=0.0002, \omega=0.001$, and $A$ is 200,500 , and 800 , respectively, the corresponding probability density, 


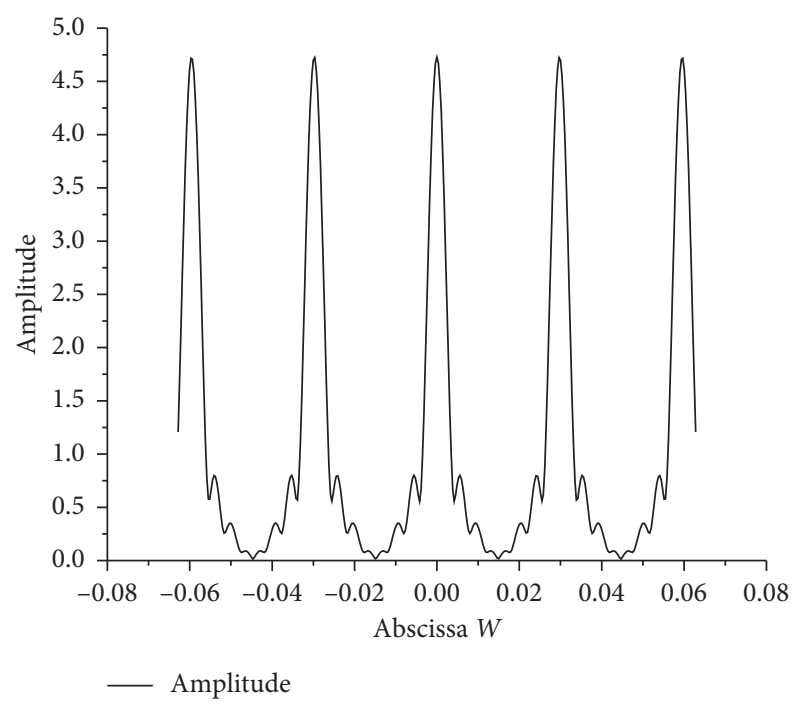

Figure 9: Empirical distribution cumulative failure probability amplitude frequency diagram.

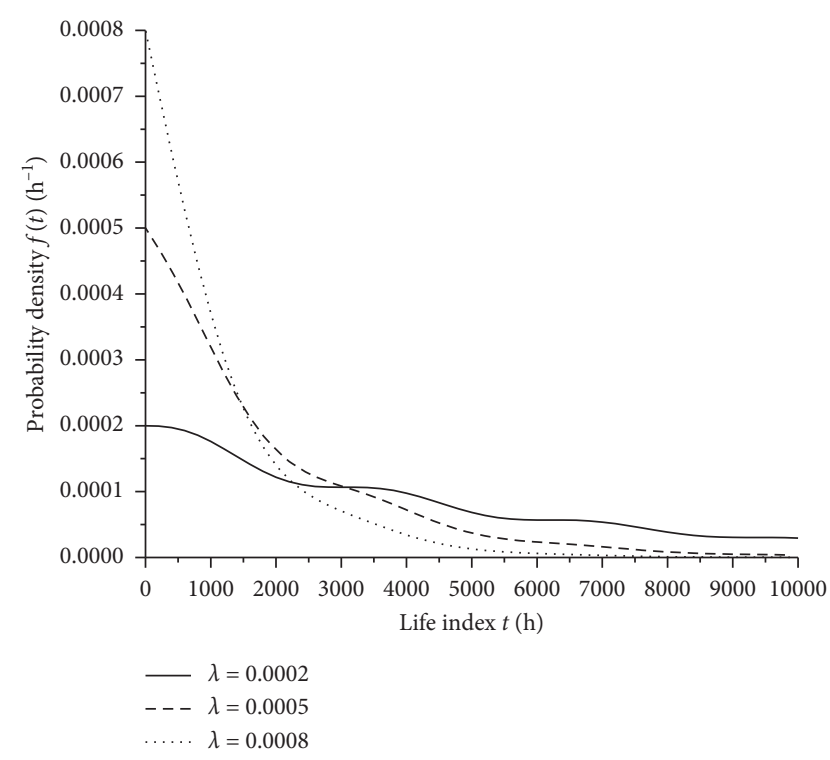

FIGURE 10: Effects of attenuation coefficient on probability density.

reliability, and failure rate sensitivity changes with respect to the oscillation angular frequency $2 \omega$ are shown in Figures 28-30.

It can be seen from Figure 31 that the larger the oscillation amplitude $A$, the smaller the fluctuation of the sensitivity curve of the probability density with respect to the oscillation angular frequency $2 \omega$ and the shorter the period. Figure 28 illustrates that the larger the oscillation amplitude $A$ is, the smaller the fluctuation of the sensitivity variation curve of reliability with respect to the oscillation angular frequency $2 \omega$ is and the larger the value is. Figure 29 shows that the sensitivity change curve of the failure rate with respect to the oscillation angular frequency $2 \omega$ has periodicity. The larger the oscillation amplitude is, the larger the

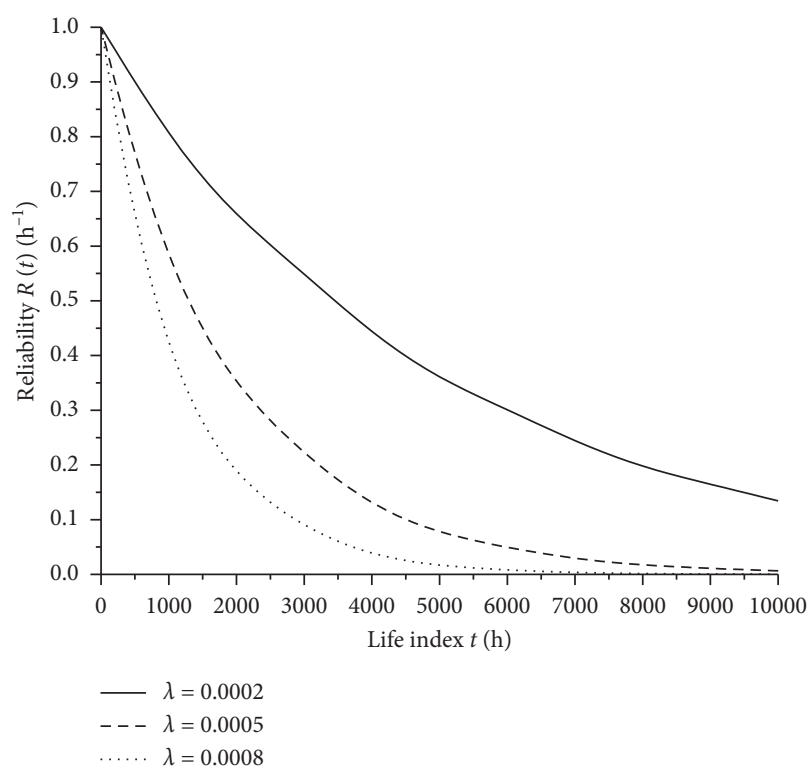

Figure 11: Effects of attenuation coefficient on reliability.

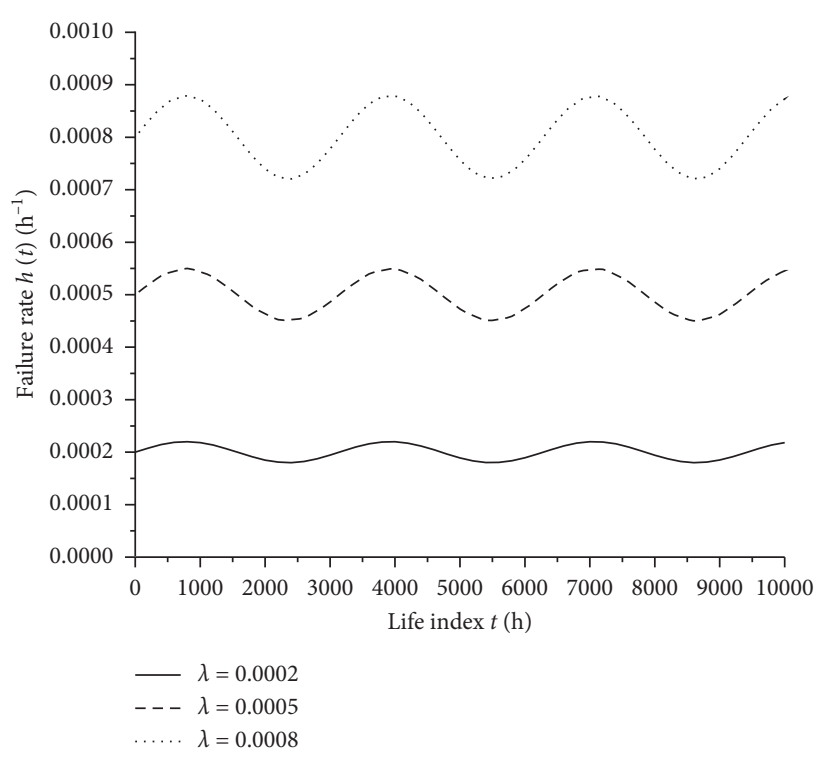

Figure 12: Effects of attenuation coefficient on failure rate.

fluctuation is and the period remains unchanged; afterwards, the fluctuation will become increasingly greater.

5.4. The Influence of Oscillation Angular Frequency on the Sensitivity of Life Probability Distribution Characteristics. When $\lambda=0.0002, A=500$, and $\omega$ is $0.0002,0.000$, and 0.0006 , respectivel $y$, the corresponding probability density, reliability, and failure rate with respect to the sensitivity change curve of the attenuation coefficient $\lambda$ are shown in Figures 32-34.

It can be seen from Figures 30, 32, and 33 that the greater the oscillation angular frequency $\omega$ is, the greater the fluctuation of the sensitivity curve of probability density, reliability, and failure rate with respect to the attenuation 


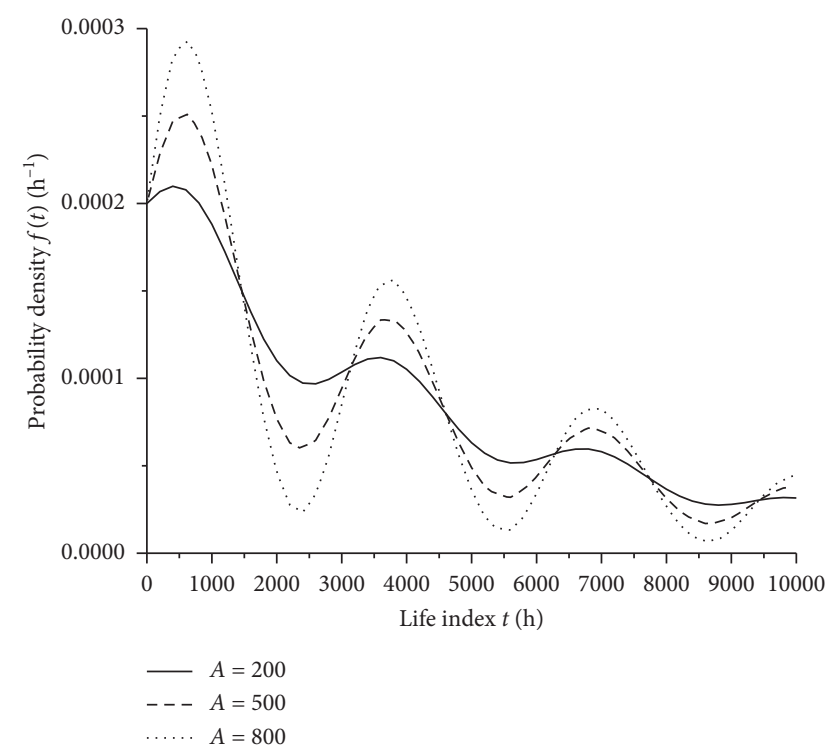

FIGURE 13: Effects of oscillation amplitude on probability density.

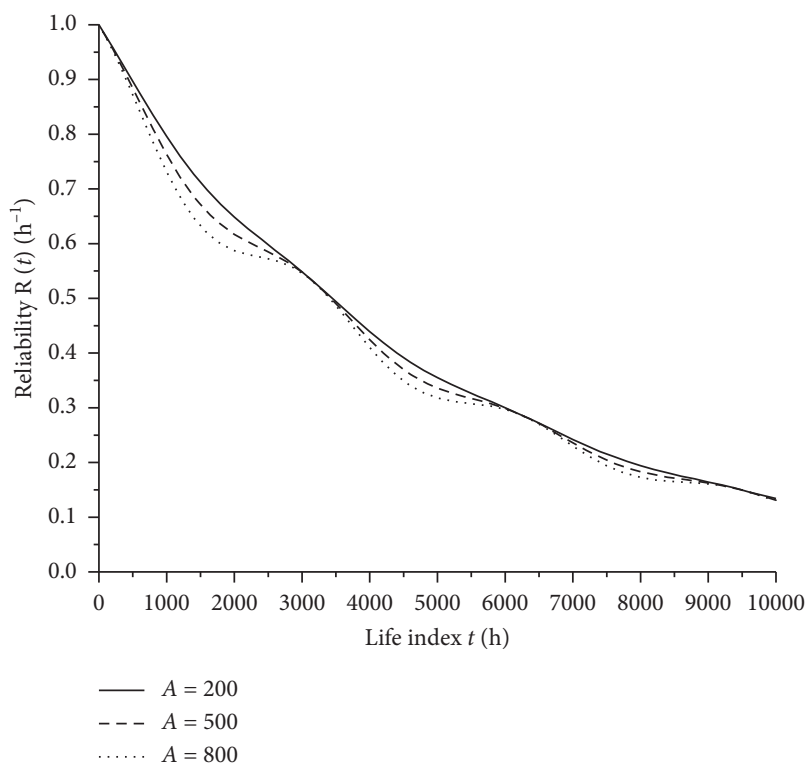

Figure 14: Effects of oscillation amplitude on reliability.

coefficient $\lambda$ is. Meanwhile, it can be seen from Figures 30 and 32 that the trend of curve change of the two graphs is similar, with only slight numerical differences. Figure 33 shows that the sensitivity change curve of the failure rate with respect to the attenuation coefficient $\lambda$ has periodicity, and the larger the oscillation angular frequency $\omega$ is, the shorter the period is and the larger the amplitude is.

When $\lambda=0.0002, A=500$, and $\omega$ is $0.0002,0.0004$, and 0.0006 , respectively, the corresponding probability density, reliability, and failure rate with respect to the sensitivity variation curve of the oscillation amplitude $A$ are shown in Figures 34-36.

It can be seen from Figures 34-36 that the greater the oscillation angular frequency $\omega$ is, the greater the fluctuation of the sensitivity curve of probability density, reliability, and

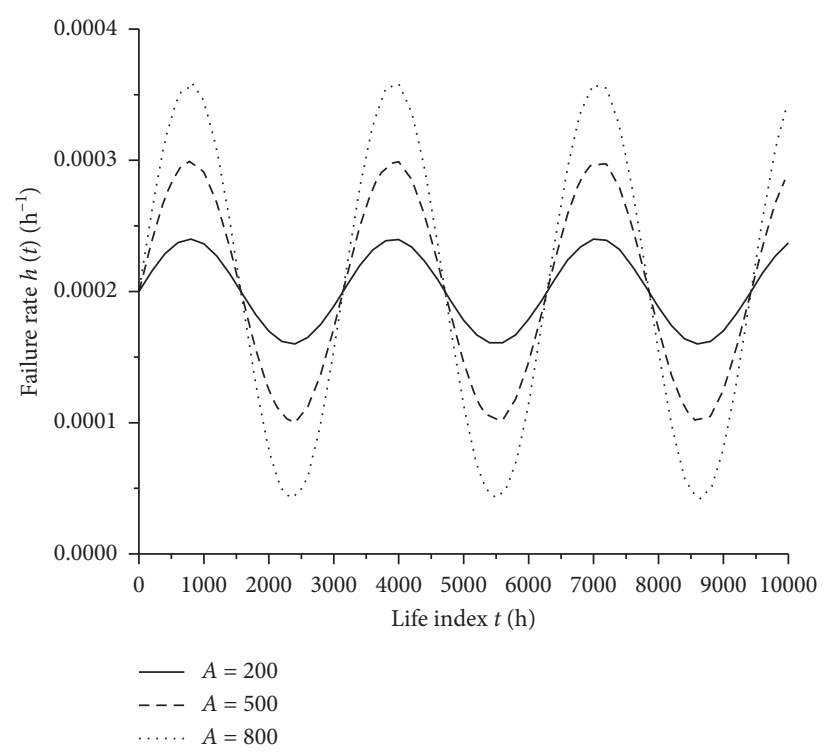

FIgURE 15: Effects of oscillation amplitude on failure rate.

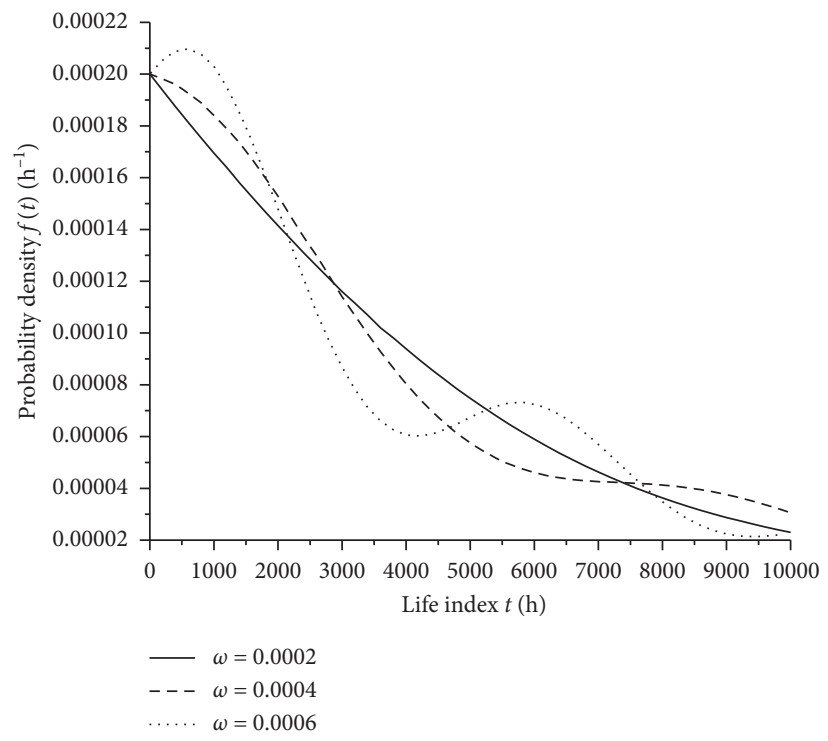

FIGURE 16: Effects of oscillation angular frequency on probability density.

failure rate with respect to the oscillation amplitude $A$ is. At the same time, it can be seen from Figures 34 and 35 that the sensitivity variation curve of probability density and reliability with respect to the oscillation amplitude $A$ fluctuates greatly at the initial position and tends to be smoother later. As can be seen from Figure 36, the sensitivity change curve of the failure rate with respect to the oscillation amplitude $A$ is periodic. The larger the oscillation angular frequency $\omega$ is, the shorter the period is and the larger the amplitude is.

\section{Model Parameter Estimation Based on Maximum Likelihood}

Suppose $T_{1}, T_{2}, \ldots, T_{n}$ are samples from an ensemble $T$ with a capacity of $n$, and $t_{1}, t_{2}, \ldots, t_{n}$ are the sample value 


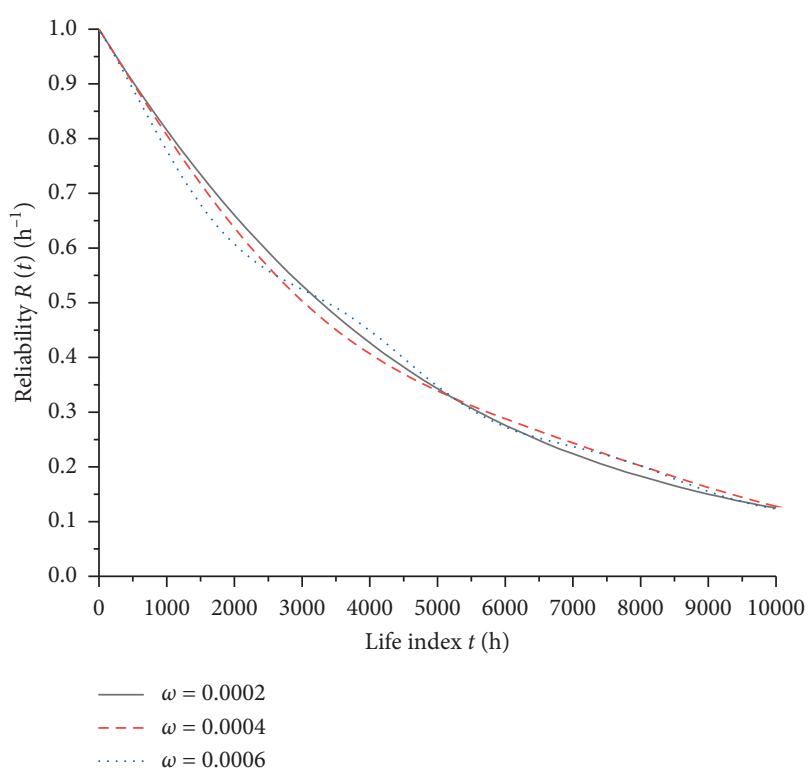

FIGURE 17: Effects of oscillating angular frequency on reliability.

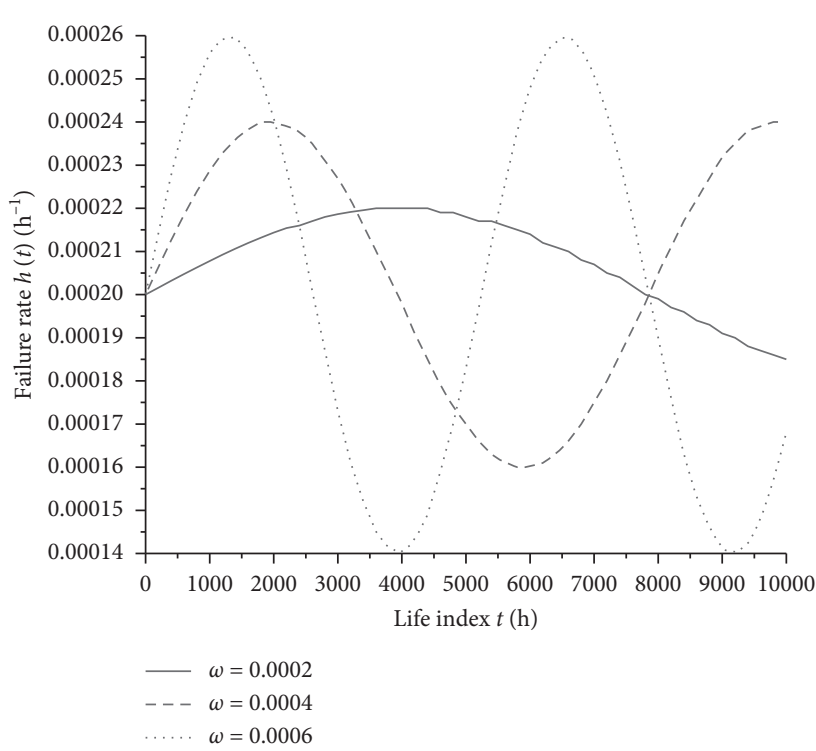

FIGURE 18: Effects of oscillation angular frequency on failure rate.

corresponding to sample $T_{1}, T_{2}, \ldots, T_{n}$. The likelihood function corresponding to formula (1) is

$$
\begin{aligned}
& L\left(t_{1}, t_{2}, \ldots, t_{n} ; \lambda, A, \omega\right) \\
& \quad=\prod_{i=1}^{n} \lambda\left[A \omega \sin \left(2 \omega t_{i}\right)+1\right] e^{-\lambda\left[A \sin ^{2}\left(\omega t_{i}\right)+t_{i}\right]} .
\end{aligned}
$$

Equation (11) calculates the partial derivatives of $\lambda, A, \omega$, respectively; then,

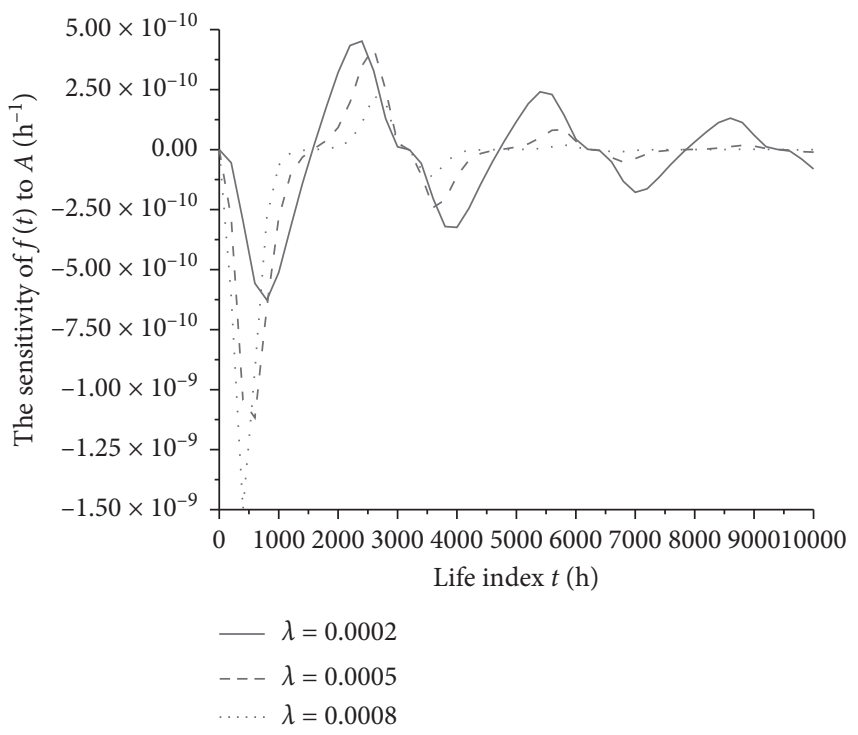

FIgURE 19: The sensitivity of $f(t)$ to $A(\lambda=0.0002 / 0.0005 / 0.0008)$.

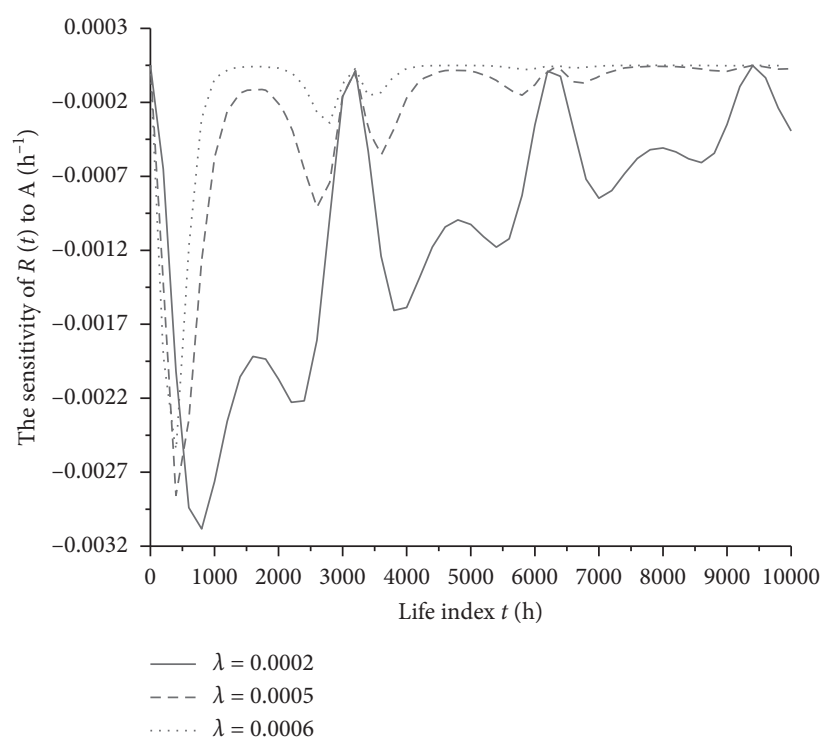

Figure 20: The sensitivity of $R(t)$ to $A(\lambda=0.0002 / 0.0005 / 0.0008)$.

$$
\frac{\partial \ln L}{\partial \lambda}=n-\sum_{i=1}^{n}\left[A \sin ^{2}\left(\omega t_{i}\right)+t_{i}\right]=0,
$$

$$
\frac{\partial \ln L}{\partial A}=\sum_{i=1}^{n} \frac{\omega \sin \left(2 \omega t_{i}\right)}{A \omega \sin \left(2 \omega t_{i}\right)+1}-\lambda \sum_{i=1}^{n} \sin ^{2}\left(\omega t_{i}\right)=0,
$$

$$
\begin{aligned}
\frac{\partial \ln L}{\partial \omega} & =\sum_{i=1}^{n} \frac{\sin \left(2 \omega t_{i}\right)+2 \omega t_{i} \cos \left(2 \omega t_{i}\right)}{A \omega \sin \left(2 \omega t_{i}\right)+1}-\lambda \sum_{i=1}^{n} t_{i} \sin \left(2 \omega t_{i}\right) \\
& =0 .
\end{aligned}
$$




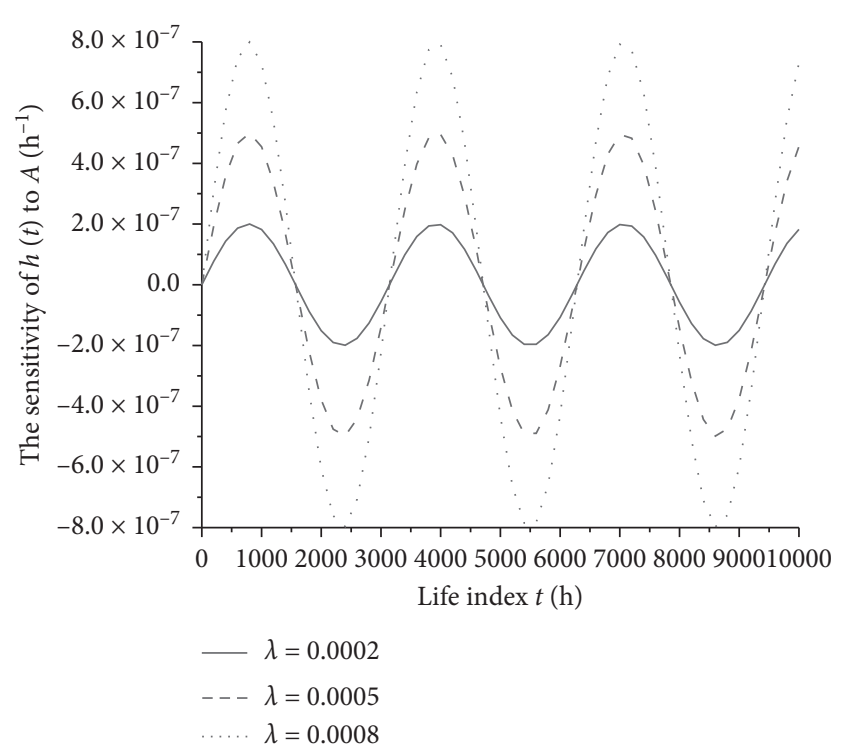

Figure 21: The sensitivity of $h(t)$ to $A(\lambda=0.0002 / 0.0005 / 0.0008)$.

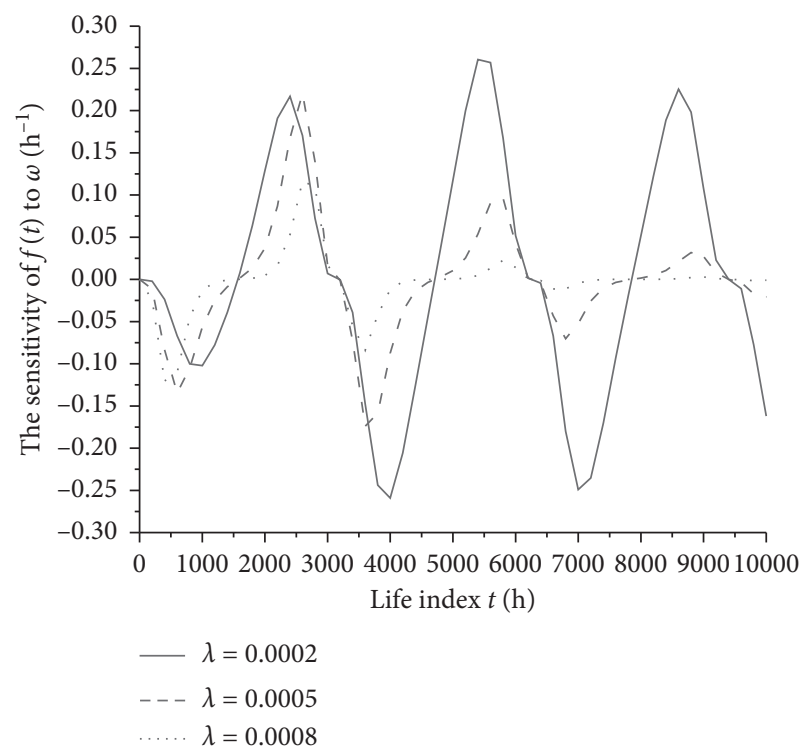

Figure 22: The sensitivity of $f(t)$ to $2 \omega(\lambda=0.0002 / 0.0005 / 0.0008)$.

By using MATLAB program, $\lambda, A, \omega$ can be obtained from successive formulas (22)-(24).

\section{Prediction of Hydraulic Automatic Transmission Reliability Based on Exponential Decay Oscillation Distribution Model}

Based on the failure data of the hydraulic automatic gearbox at several points in time (Table 1), the life probability density value is calculated. Combining the life probability density and reliability of the hydraulic automatic gearbox, the

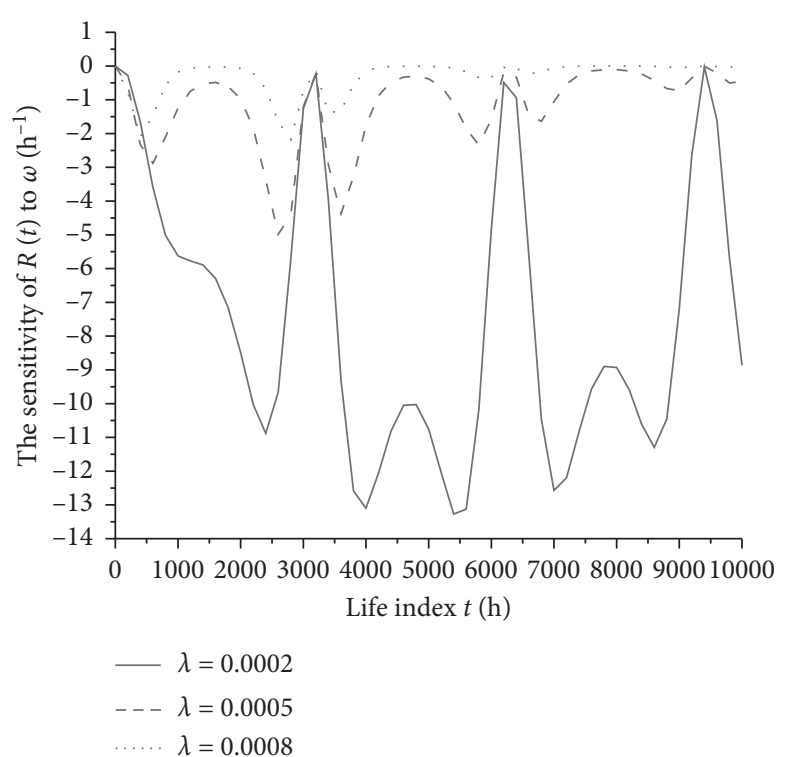

Figure 23: The sensitivity of $R(t)$ to $2 \omega(\lambda=0.0002 / 0.0005 / 0.0008)$.

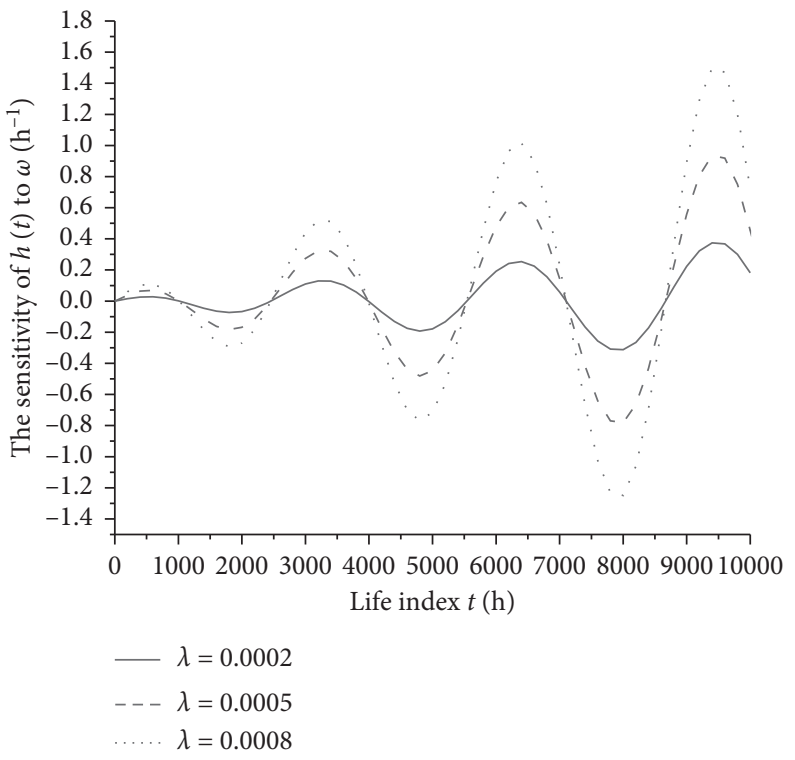

Figure 24: The sensitivity of $h(t)$ to $2 \omega(\lambda=0.0002 / 0.0005 / 0.0008)$.

variation characteristics of attenuated oscillation, and the empirical distribution of the cumulative failure probability of the amplitude frequency spectrum with the increase of time, it is concluded that it has an obvious characteristic frequency. On this basis, formula (1) is used to perform parameter fitting on its life probability density characteristics and compared with the fitting results of exponential distribution, three-parameter Weibull, and "bathtub curve" model proposed in [54]. The comparing results are shown in Figure 37, and the root mean square error of the fitting results of the four methods is shown in Table 2. 


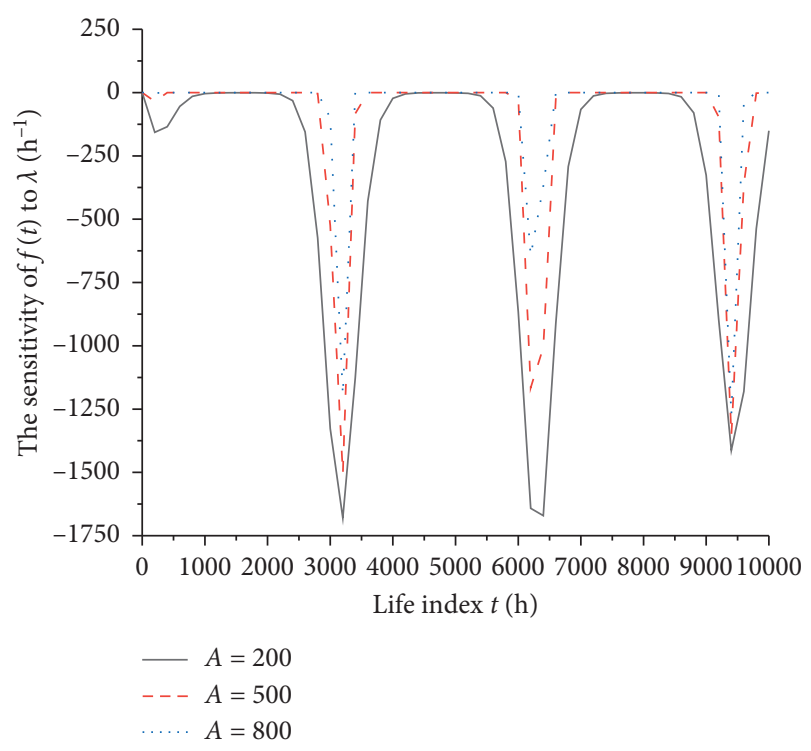

FIgure 25: The sensitivity of $f(t)$ to $\lambda(A=200 / 500 / 800)$.

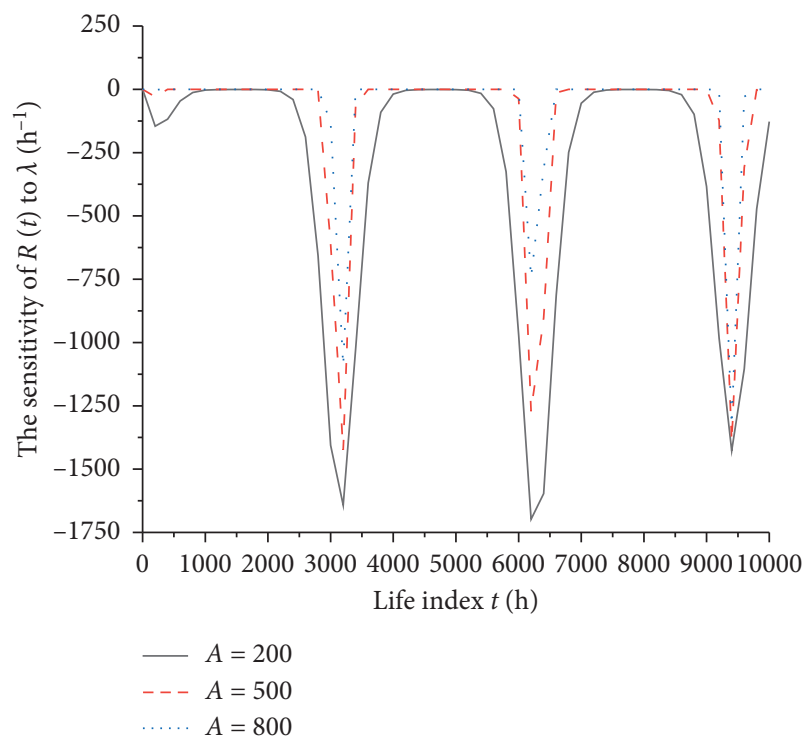

Figure 26: The sensitivity of $R(t)$ to $\lambda(A=200 / 500 / 800)$.

As can be seen from Figure 37 and Table 2, compared with the exponential distribution, the three-parameter Weibull distribution, and the "bath curve" model proposed in [52], the exponential decay oscillation model established in the shape of the fitted curve can better simulate the attenuation oscillation characteristics of the fault-free working time probability density of the hydraulic automatic transmission at $t$ of $528.28 \mathrm{~h}, 739.32 \mathrm{~h}, 950.35 \mathrm{~h}, 1161.38 \mathrm{~h}$, and $1372.42 \mathrm{~h}$. This is because, in the decay oscillation model, the exponential part

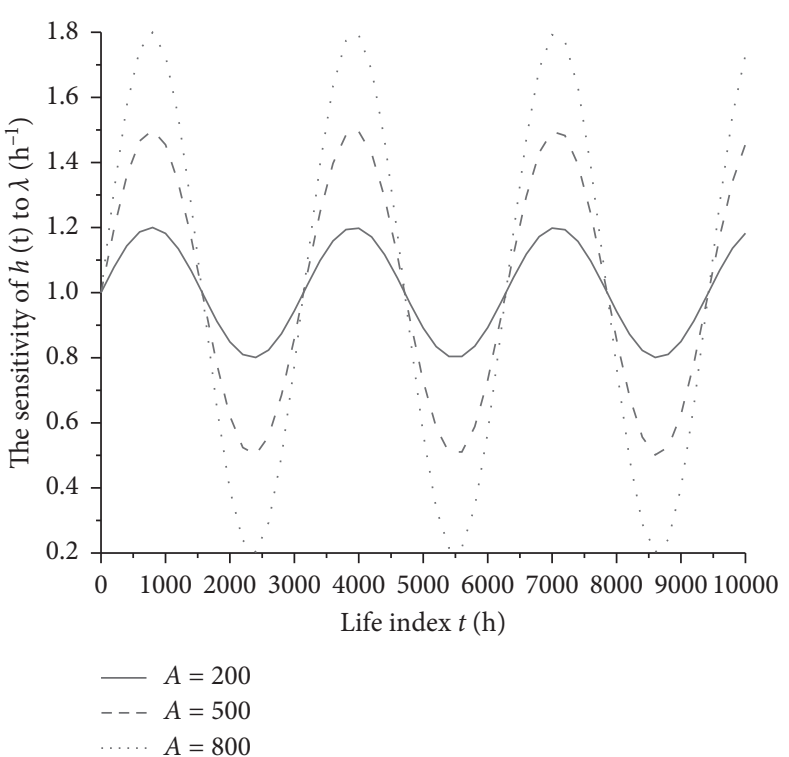

FIgURE 27: The sensitivity of $h(t)$ to $\lambda(A=200 / 500 / 800)$.

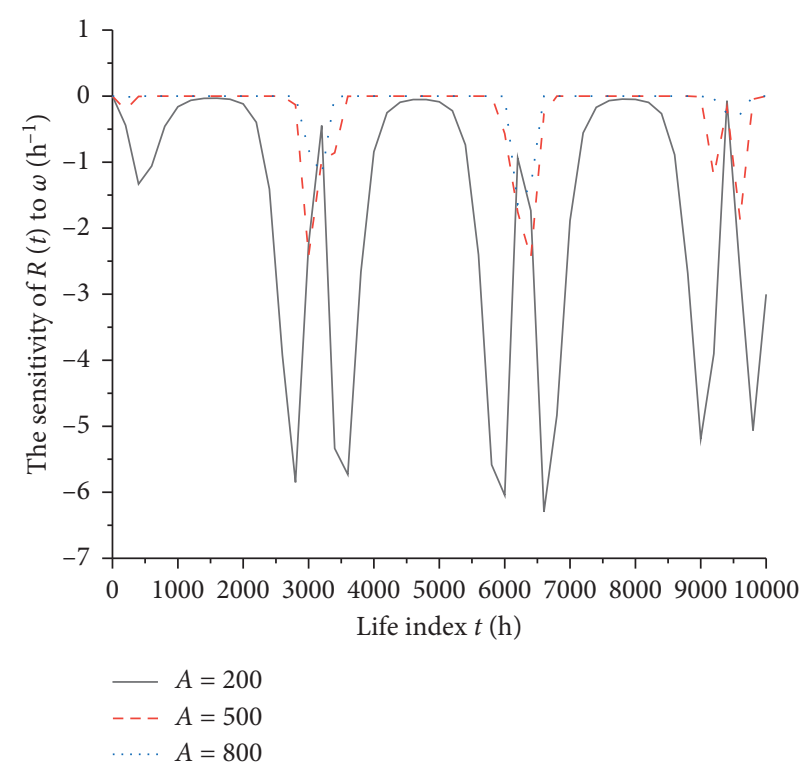

Figure 28: The sensitivity of $R(t)$ to $2 \omega(A=200 / 500 / 800)$.

introduces a periodic function to better describe the oscillation characteristics of the data. Meanwhile, as can be seen from Table 2, the exponential decay oscillation model can obtain a smaller fitted mean square root error.

With the probability density model shown in equation (1), the probability density fitting model of the troublefree working time of the hydraulic automatic transmission is calculated as 


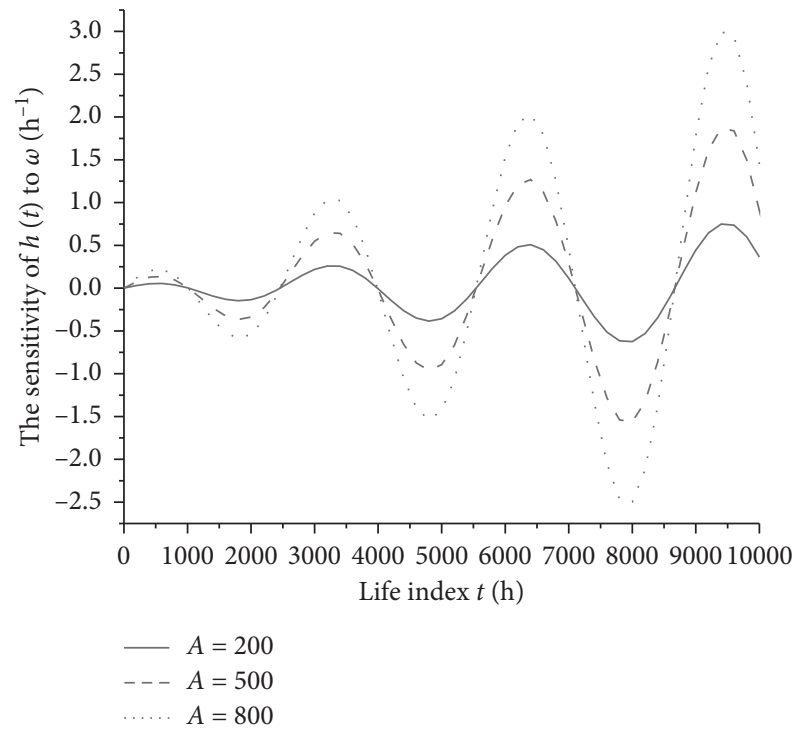

Figure 29: The sensitivity of $h(t)$ to $2 \omega(A=200 / 500 / 800)$.

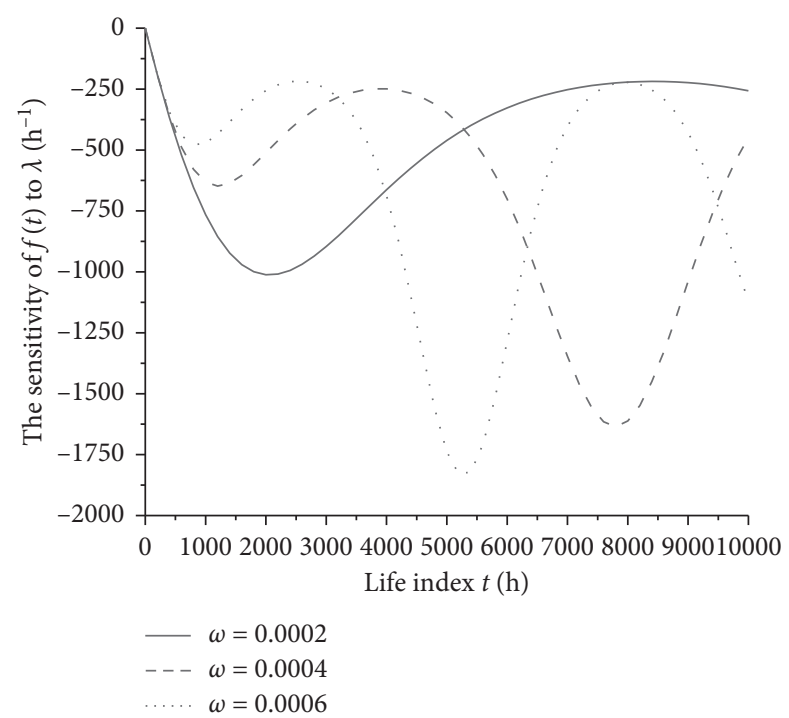

FIgURE 30: The sensitivity of $f(t)$ to $\lambda(\omega=0.0002 / 0.0004 / 0.0006)$.

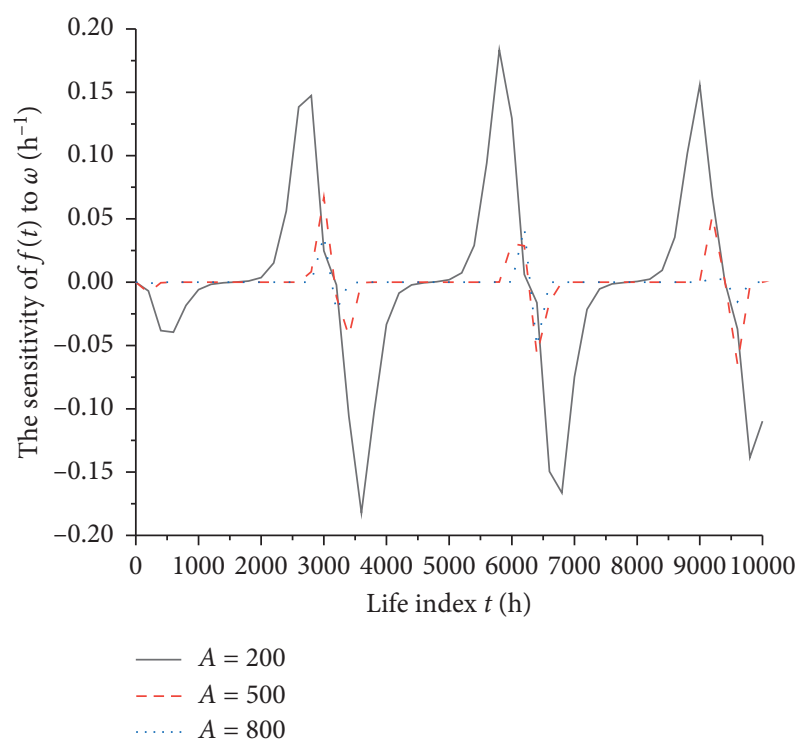

Figure 31: The sensitivity of $f(t)$ to $2 \omega(A=200 / 500 / 800)$. 


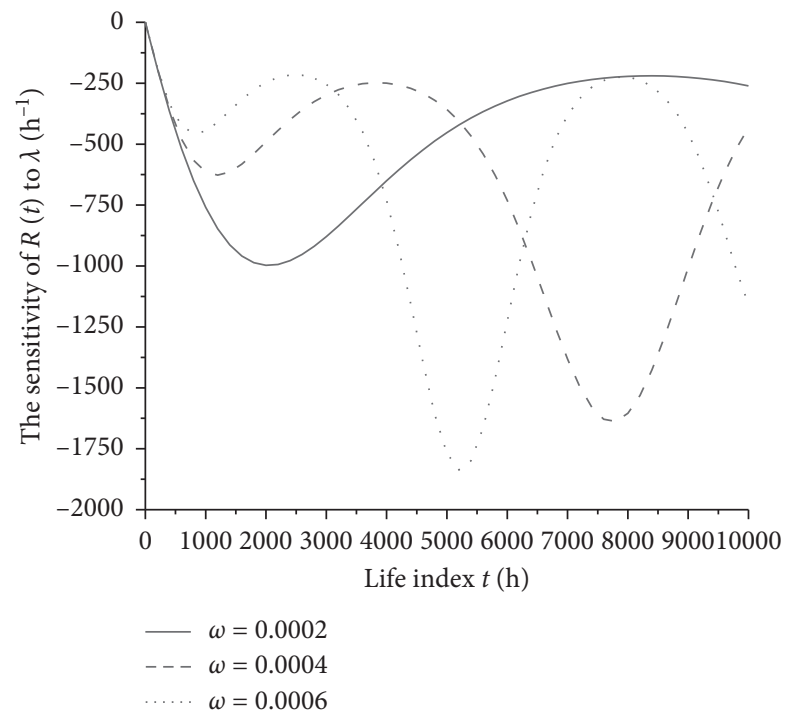

Figure 32: The sensitivity of $R(t)$ to $\lambda(\omega=0.0002 / 0.0004 / 0.0006)$.

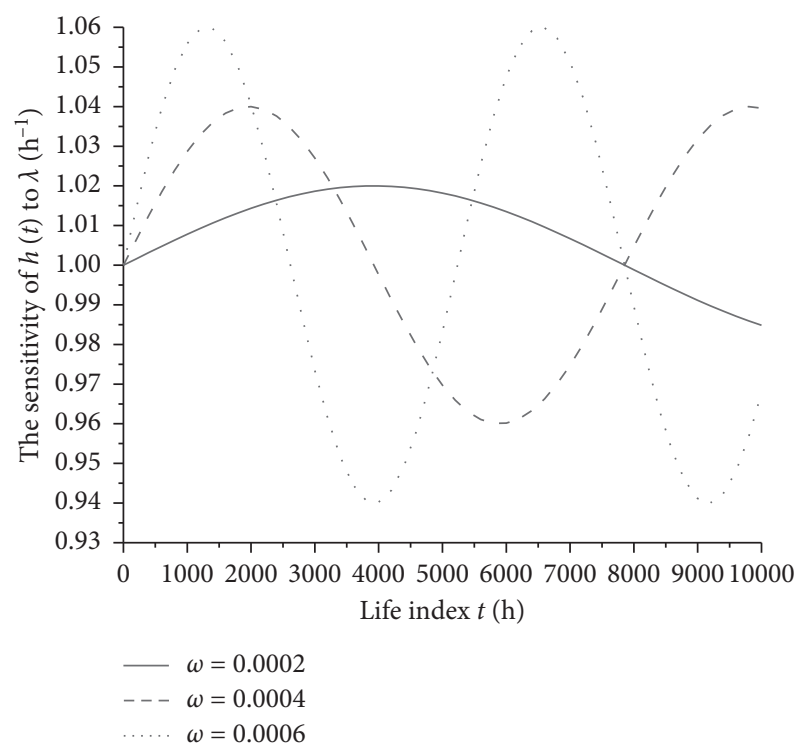

Figure 33: The sensitivity of $h(t)$ to $\lambda(\omega=0.0002 / 0.0004 / 0.0006)$.

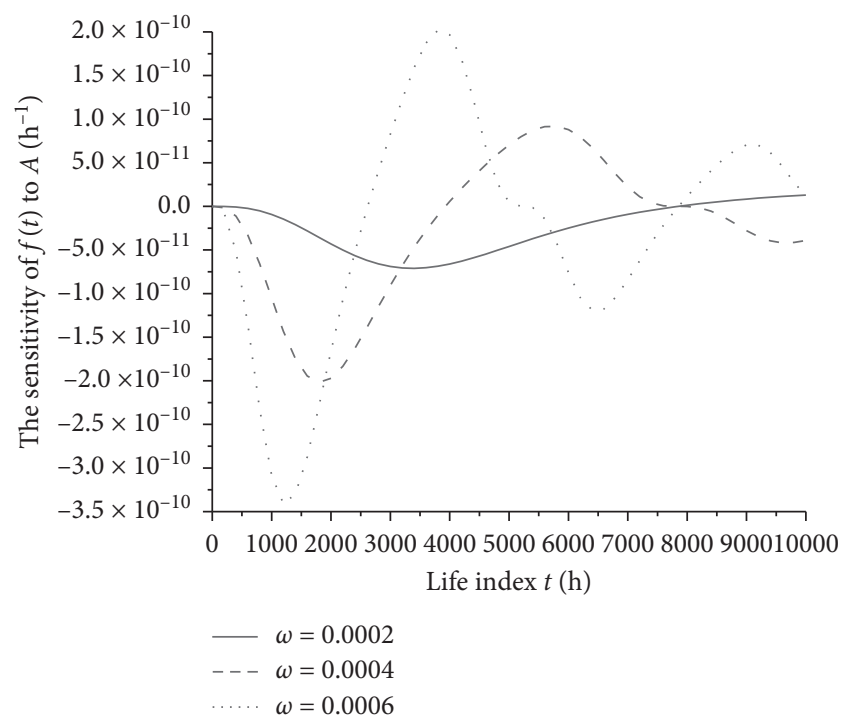

Figure 34: The sensitivity of $f(t)$ to $A(\omega=0.0002 / 0.0004 / 0.0006)$. 


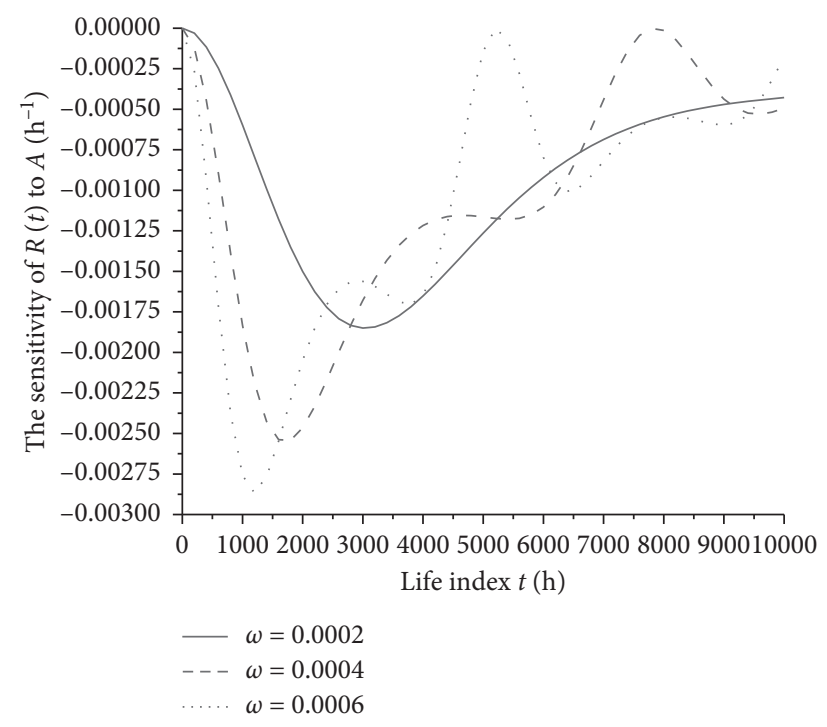

Figure 35: The sensitivity of $R(t)$ to $A(\omega=0.0002 / 0.0004 / 0.0006)$.

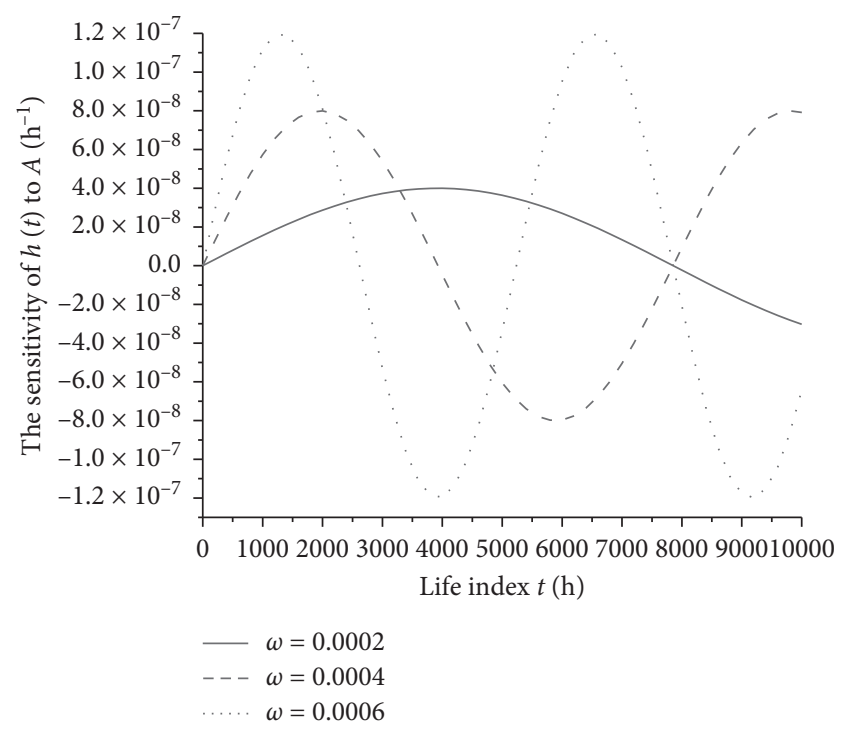

FIgURE 36: The sensitivity of $h(t)$ to $A(\omega=0.0002 / 0.0004 / 0.0006)$.

$$
\begin{aligned}
f(t)= & \frac{9.818}{211.03} \times 10^{-4} \\
& \times\left[5.534 \times 10^{-2} \sin \left(1.134 \times 10^{-2} t\right)+1\right] \\
& \times e^{-9.818 \times 10^{-4} \times\left[-9.407 \sin ^{2}\left(5.67 \times 10^{-3} t\right)+t\right]} .
\end{aligned}
$$

Furthermore, the reliability and failure rate models can be obtained as

$$
\begin{aligned}
& R(t)=e^{-9.818 \times 10^{-4} \times\left[-9.407 \sin ^{2}\left(5.67 \times 10^{-3} t\right)+t\right]}, \\
& h(t)=\frac{9.818}{211.03} \times 10^{-4} \times\left[5.534 \times 10^{-2} \sin \left(1.134 \times 10^{-2} t\right)+1\right] .
\end{aligned}
$$




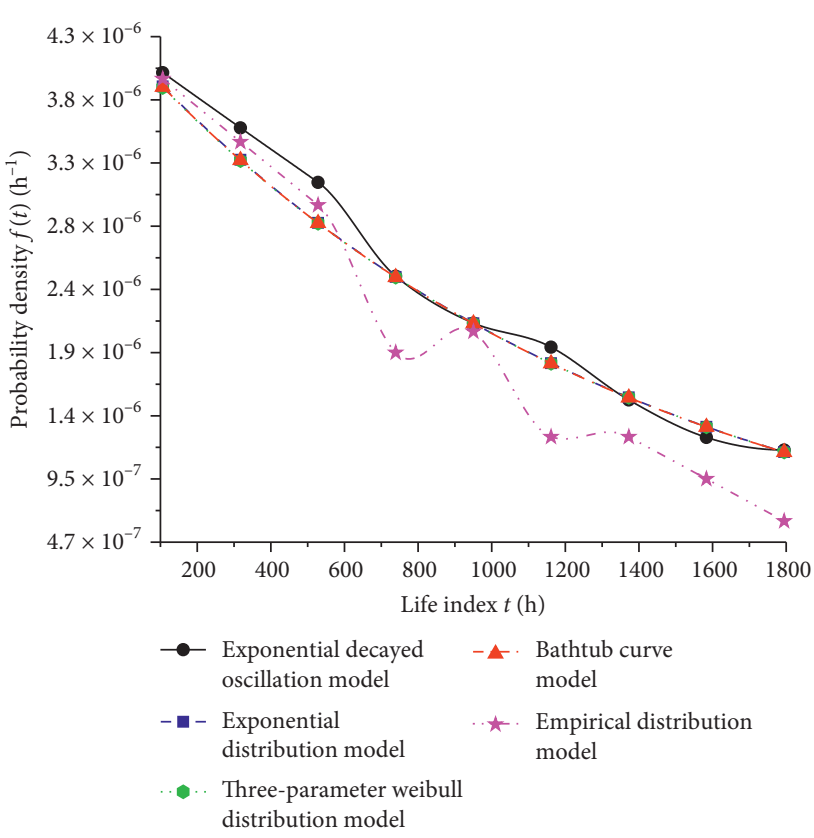

Figure 37: Comparison of probability density.

TABLE 2: Comparison of root mean square error.

\begin{tabular}{lc}
\hline Probability density model & $\begin{array}{c}\text { Root mean square error of the } \\
\text { fitting results }\end{array}$ \\
\hline $\begin{array}{l}\text { Exponential decay oscillation } \\
\text { model }\end{array}$ & $3.677 e-005$ \\
$\begin{array}{l}\text { Exponential distribution model } \\
\text { Three-parameter Weibull }\end{array}$ & $5.318 e-005$ \\
distribution model & $5.783 e-005$ \\
Bathtub curve model & $7.052 e-005$ \\
\hline
\end{tabular}

\section{Conclusion}

(1) The empirical distribution model is used to judge the reliability statistical characteristics of a certain type of hydraulic automatic transmission. On this basis, the Fourier analysis method is used to judge the empirical distribution statistical characteristics of the transmission reliability. It is concluded that the reliability of the gearbox has the characteristics of periodic attenuation and oscillation. At the same time, this method provides meaningful guidance and reference for analyzing the characteristics of the product life distribution model.

(2) Based on the exponential cumulative failure distribution model, the reliability model of exponential decay oscillation distribution is constructed by introducing a periodic function $A \sin ^{2}(\omega t)$ with oscillation characteristics, and the corresponding probability density, reliability, failure rate, and average life calculation models are derived.

(3) On this basis, the influence of attenuation coefficient, oscillation amplitude, oscillation angle frequency, and other parameters on the characteristics of probability distribution is studied. On balance, the probability density exhibits an exponentially declining oscillation change law, and the failure rate is no longer an invariant constant but has certain periodicity and volatility. Among them, the smaller the attenuation coefficient $\lambda$ is, the smaller the failure rate is, the higher the reliability is, and the gentler the change in probability density is; the greater the oscillation amplitude $A$ is, the more severe the oscillations of probability density, reliability, and failure rate are; the greater the oscillation angle frequency $\omega$ is, the faster the oscillations of probability density, reliability, and failure rate are.

(4) The empirical distribution probability density is fitted by the exponential decay oscillation probability density model and compared with the fitting results of exponential distribution, three parameters Weibull distribution, and "bathtub curve" model established in [52]. The comparison results show that the exponential decay oscillation model can better simulate the attenuation oscillation characteristics of the probability density of trouble-free working time of the hydraulic automatic transmission. Using the exponential decay oscillation model, the fitting root mean square error is $3.677 e-005$.

The research ideas and conclusions of this paper enrich the reliability life distribution model. Further research will be carried out not only on the reliability of the model in the fields of optoelectronics, aviation, aerospace, and other types of nonelectronic equipment but also on the adaptability in its engineering application. In addition, the research objects will expand from the component and device level to equipment, subsystems, and systems.

\section{Data Availability}

The data used to support the findings of this study are included within the article.

\section{Conflicts of Interest}

The authors declare that they have no conflicts of interest.

\section{Acknowledgments}

This paper belongs to the major projects of the Anhui Province Natural Science Foundation (1808085ME127), the Anhui Polytechnic University Research Initiation Fund for Introducing Talents (2019YQQ004), the Anhui Polytechnic University Research Project (Xjky019201905), the Industrial Collaborative Innovation Fund of Anhui Polytechnic University and Jiujiang District (2021cyxtb9), and the Open Project of Anhui Provincial Engineering Laboratory on Information Fusion and Control of Intelligent Robot (IFCIR2020001).

\section{References}

[1] L. M. Clement, "Reliability of military electronic equipment," Journal of the British Institution of Radio Engineers, vol. 16, no. 9, pp. 488-495, 1956. 
[2] C. Borzea and C. D. Comeaga, "Reliability of Euler-Bernoulli model for multilayer composite piezoelectric beams," in Proceedings of the 11th International Symposium on Advanced Topics in Electrical Engineering, Bucharest, Romania, 2019.

[3] L. I. Kunfeng and Y. Zichun, "Info-gap reliability model for structures with introduced fuzzy parameter," Journal of Huazhong University of Science and Technology (Natural Science Edition), vol. 47, 2019.

[4] Y. Qiao, W. Xu, J. Sun, and H. Zhang, "Reliability of electrostatically actuated MEMS resonators to random mass disturbance," Mechanical Systems and Signal Processing, vol. 121, no. 15, pp. 711-724, 2019.

[5] L. K. Yu, S. Im, C. S. Lau et al., System and Method to Enhance Solder Joint Reliability, United States Patent and Trademark Office, Western Digital Technologies, Inc., San Jose, CA, USA, 2019.

[6] B. Liao, B. Sun, Y. Li et al., "Sealing reliability modeling of aviation seal based on interval uncertainty method and multidimensional response surface," Chinese Journal of Aeronautics, vol. 32, no. 9, 2019.

[7] H. Liu, Y. Sun, G. L. Chen et al., "Online short-term reliability evaluation using a fast sorting technique," IET Generation, Transmission \& Distribution, vol. 2, no. 1, pp. 139-148, 2008.

[8] H. Liu, L. Cheng, Y. Sun et al., "Outage factors analysis and outage rate model of components based on operating conditions," Automation of Electric Power Systems, vol. 31, no. 7, p. 44, 2007, in Chinese.

[9] Y. Sun, L. Cheng, and H. Liu, "Power system operating reliability evaluation based on real-time operating state," Power System Technology, vol. 29, no. 15, pp. 6-12, 2005, in Chinese.

[10] Y. Sun, X. Wang, J. Wang, and S. Xie, "An overview of the short-term reliability evaluation of power system," Power System Protection and Control, vol. 39, no. 8, pp. 143-154, 2011.

[11] R. Billinton and G. Singh, "Application of adverse and extreme adverse weather: modeling in transmission and distribution system reliability evaluation," IEEE ProceedingsGeneration, Transmission and Distribution, vol. 153, no. 1, pp. 115-120, 2006.

[12] W. Li, J. Zhou, K. Xie et al., "Power system risk assessment using a hybrid method of fuzzy set and Monte Carlo simulation," IEEE Transactions on Power Systems, vol. 23, no. 2, pp. 336-343, 2008.

[13] R. Nateghi, "Multi-dimensional infrastructure resilience mode-ling: an application to hurricane-prone electric power distribution systems," IEEE Access, vol. 6, pp. 13478-13489, 2018.

[14] K. F. Tee and K. Pesinis, "Reliability prediction for corroding natural gas pipelines," Tunneling and Underground Space Technology, vol. 65, pp. 91-105, 2017.

[15] M. Valinčius, I. Žutautaitè, G. Dundulis et al., "Integrated assessment of failure probability of the district heating network," Reliability Engineering \& System Safety, vol. 133, pp. 314-322, 2015.

[16] X. Wang, Study on Data-Driven Fault Diagnosis for Proton Exchange Membrane Fuel Cell Stack, Southwest Jiaotong University, Chengdu, China, 2018, in Chinese.

[17] B. Gui, The Study of Centrifugal Compressor Fault Diagnosis Based on the Ant Colonies Algorithm, Xi' an Shiyou University, Xi'an, China, 2015, in Chinese.

[18] Y. Ding, Y. Jiang, Y. Song et al., "Review of risk assessment for energy internet, part I: physical level," Proceeding of the CSEE, vol. 36, no. 14, pp. 3806-3817, 2016, in Chinese.
[19] L. Xie, "Issues and commentary on mechanical reliability theories, methods and models," Journal of Mechanical Engineering, vol. 50, no. 14, pp. 27-35, 2014, in Chinese.

[20] G. H. Yang, S. Si-Il, and K. Y. Soo, "Prediction of system reliability using failure types of components based on Weibull lifetime distribution," Journal of Mechanical Science and Technology, vol. 32, no. 6, pp. 2463-2471, 2018.

[21] W. Weibull, "A statistical distribution function of wide applicability," Journal of Applied Mechanics, vol. 18, pp. 293297, 1951.

[22] A. J. Hallinan Jr., "A review of the Weibull distribution," Journal of Quality Technology, vol. 25, no. 2, pp. 85-93, 1993.

[23] G. M. Cordeiro, E. M. M. Ortega, and A. J. Lemonte, "The exponential-Weibull lifetime distribution," Journal of Statistical Computation and Simulation, vol. 84, no. 12, pp. 2592-2606, 2014.

[24] F. Wang, X. Chen, B. Dun, B. Wang, D. Yan, and H. Zhu, "Rolling bearing reliability assessment via kernel principal component analysis and Weibull proportional hazard model," Shock and Vibration, vol. 2017, Article ID 6184190, 11 pages, 2017.

[25] J. K. Freels, D. A. Timme, J. J. Pignatiello, R. L. Warr, and R. R. Hill, "Maximum likelihood estimation for the polyWeibull distribution," Quality Engineering, vol. 31, no. 4, pp. 545-552, 2019.

[26] J. E. Baloui, “Analyzing system reliability using fuzzy Weibull lifetime distribution," International Journal of Applied Operational Research, vol. 4, no. 1, pp. 93-102, 2014.

[27] D. Nan, Z.-x. Jia, and L. I. Wei, "Monte Carlo based parametric point estimation for three parameter Weibull distribution," Machinery Design \& Manufacture, no. 1, pp. 142-144, 2017, in Chinese.

[28] W. Linying, H. Hu, and W. Wu, "Operational reliability modeling of complex electronic systems based on exponential Weibull distribution," Journal of Detection \& Control, vol. 41, no. 2, pp. 115-119, 2019, in Chinese.

[29] L. C. M. González, M. I. R. Borbón, D. J. Valles-Rosales, A. D. Valle, and A. Rodriguez, "Reliability model for electronic devices under time varying voltage," Quality and Reliability Engineering International, vol. 32, no. 4, 2016.

[30] L. C. Mendez-Gonzalez, M. Iván Rodríguez-Borbón, M. R. Piña-Monarrez, R. Ambrosio, and A. Valle, "Reliability analysis for laptop computer under electrical harmonics," Quality and Reliability Engineering International, vol. 32, no. 8, 2016.

[31] G. Ling, Y. Dai, and X. Li, "Maximum entropy prior information solution based on second moment and its application in NC system reliability assessment," China Mechanical Engineering, vol. 21, no. 12, pp. 1466-1468, 2010, in Chinese.

[32] L. Gao, W. Chen, P. Qian, J. Pan, and Q. He, "Optimal timecensored constant-stress ALT plan based on chord of nonlinear stress-life relation- ship," IEEE Transactions on Reliability, vol. 65, no. 3, pp. 1496-1508, 2016.

[33] S. Ephraim, "Could electronics reliability be predicted, quantified and assured?" Microelectronics Reliability, vol. 53, no. 7, pp. 925-936, 2013.

[34] P. Qian, "Research of multiple stresses accelerated life model verification method," Journal of Mechanical Engineering, vol. 46, no. 24, pp. 156-161, 2010.

[35] J. I. Park and S. J. Bae, "Direct prediction methods on lifetime distribution of organic light-emitting diodes from accelerated degradation tests," IEEE Transactions on Reliability, vol. 59, no. 1, pp. 74-90, 2010. 
[36] H. Liao and E. A. Elsayed, "Reliability inference for field conditions from accelerated degradation testing," Naval Research Logistics (NRL), vol. 53, no. 6, 2006.

[37] C.-M. Liao and S.-T. Tseng, "Optimal design for step-stress accelerated degradation tests," IEEE Transactions on Reliability, vol. 55, no. 1, pp. 59-66, 2006.

[38] S. Hao, J. Yang, and C. Berenguer, "Nonlinear step-stress accelerated degradation modelling considering three sources of variability," Reliability Engineering \& System Safety, vol. 172, pp. 207-215, 2017.

[39] S. Shemehsavar and M. Amini, "Failure inference and optimization for step stress model based on bivariate wiener model," Communications in Statistics-Simulation \& Computation, vol. 45, 2016.

[40] T.-R. Tsai, W.-Y. Sung, Y. L. Lio, S. I. Chang, and J.-C. Lu, "Optimal two-variable accelerated degradation test plan for gamma degradation processes," IEEE Transactions on Reliability, vol. 65, no. 1, pp. 459-468, 2016.

[41] P. W. Srivastava, Manisha, and M. L. Agarwal, "Design of bivariate step-stress partially accelerated degradation test plan using copula and gamma process," International Journal of Reliability and Applications, vol. 17, no. 1, pp. 21-49, 2016.

[42] W. D. Xu, An Inverse Gaussian Process Model for Degradation Data, Technimetrics Inc., New York, NY, USA, 2010.

[43] Z.-S. Ye, "On the conditional increments of degradation processes," Statistics \& Probability Letters, vol. 83, no. 11, pp. 2531-2536, 2013.

[44] Z. S. Ye and N. Chen, "The inverse Gaussian process as a degradation model," Technometrics, vol. 56, no. 3, 2014.

[45] G. Jin, Reliability Technology Based on Degradation-Model. Method and Application, National Defense Industry Press, Beijing, China, 2014.

[46] C. Park and W. J. Padgett, "Accelerated degradation models for failure based on geometric brownian motion and gamma processes," Lifetime Data Analysis, vol. 11, no. 4, pp. 511-527, 2005.

[47] X.-S. Si, W. Wang, C.-H. Hu, D.-H. Zhou, and M. G. Pecht, "Remaining useful life estimation based on a nonlinear diffusion degradation process," IEEE Transactions on Reliability, vol. 61, no. 1, pp. 50-67, 2012.

[48] D. Nouguier, G. Pananakakis, X. Federspiel, M. Rafik, D. Roy, and G. Ghibaudo, "On the cumulative distribution function of the defect centric model for BTI reliability," Microelectronics Reliability, vol. 92, pp. 168-171, 2019.

[49] D. Peng, W. Sima, M. Yang, M. Zou, Y. Zhou, and Y. Liu, “An improved centered cycle method for identifying the Preisach distribution function," IEEE Transactions on Magnetics, vol. 54, no. 11, pp. 1-5, 2018.

[50] X. Lin, Y. A. Eldemerdash, O. A. Dobre, S. Zhang, and C. Li, "Modulation classification using received signal's amplitude distribution for coherent receivers," IEEE Photonics Technology Letters, vol. 29, 2018.

[51] Z.-S. Ye, Y. Wang, K.-L. Tsui, and M. Pecht, "Degradation data analysis using wiener processes with measurement errors," IEEE Transactions on Reliability, vol. 62, no. 4, pp. 772-780, 2013.

[52] M. Marseguerra, E. Zio, and M. Cipollone, "Designing optimal degradation tests via multi-objective genetic algorithms," Reliability Engineering and System Safety, vol. 79, no. 1, 2003.

[53] Q. Li and D. B. Kececioglu, "Design of an optimal plan for an accelerated degradation test: a case study," International Journal of Quality \& Reliability Management, vol. 23, no. 4, pp. 426-440, 2013.
[54] Z. Wang, Z. Wang, and L. Xie, "Life probability distribution model of products with the bathtub-shaped failure rate changing rule," Journal of Mechanical Engineering, vol. 51, no. 24, pp. 193-200, 2015, in Chinese. 